\title{
Three-dimensional vortex organization in a high-Reynolds-number supersonic turbulent boundary layer
}

\author{
G. E. ELSINGA ${ }^{1} \dagger$, R. J. ADRIA N ${ }^{2}$, \\ B. W. VAN OUDHEUSDEN ${ }^{1}$ AND F. SCARANO \\ ${ }^{1}$ Department of Aerospace Engineering, Delft University of Technology, 2629HS Delft, The Netherlands \\ ${ }^{2}$ Department of Mechanical and Aerospace Engineering, Arizona State University, \\ Tempe, AZ85287, USA
}

(Received 5 November 2008; revised 8 September 2009; accepted 8 September 2009)

Tomographic particle image velocimetry was used to quantitatively visualize the three-dimensional coherent structures in a supersonic (Mach 2) turbulent boundary layer in the region between $y / \delta=0.15$ and 0.89 . The Reynolds number based on momentum thickness $R e_{\theta}=34000$. The instantaneous velocity fields give evidence of hairpin vortices aligned in the streamwise direction forming very long zones of lowspeed fluid, consistent with Tomkins \& Adrian (J. Fluid Mech., vol. 490, 2003, p. 37). The observed hairpin structure is also a statistically relevant structure as is shown by the conditional average flow field associated to spanwise swirling motion. Spatial low-pass filtering of the velocity field reveals streamwise vortices and signatures of large-scale hairpins (height $>0.5 \delta$ ), which are weaker than the smaller scale hairpins in the unfiltered velocity field. The large-scale hairpin structures in the instantaneous velocity fields are observed to be aligned in the streamwise direction and spanwise organized along diagonal lines. Additionally the autocorrelation function of the wallnormal swirling motion representing the large-scale hairpin structure returns positive correlation peaks in the streamwise direction (at $1.5 \delta$ distance from the DC peak) and along the $45^{\circ}$ diagonals, which also suggest a periodic arrangement in those directions. This is evidence for the existence of a spanwise-streamwise organization of the coherent structures in a fully turbulent boundary layer.

\section{Introduction}

Studies of coherent structure in the outer layer of a turbulent boundary layer have historically concentrated on hairpin-like vortices as the dominant feature in these flows (Theodorsen 1952; Head \& Bandyopadhyay 1981; Robinson 1991 amongst many others) although flow visualizations also revealed larger bulges of the size of the boundary layer thickness $\delta$ (e.g. Falco 1977). More recently, it was realized that hairpin vortices are in turn organized into packets (Adrian, Meinhart \& Tomkins 2000; Christensen \& Adrian 2001), which can be thought of as a streamwise train of hairpins. Not only do these packets occur frequently, they also contribute significantly

$\dagger$ Present address: Laboratory for Aero and Hydrodynamics, Delft University of Technology, 2628CA Delft, The Netherlands. Email address for correspondence: g.e.elsinga@tudelft.nl 
to the Reynolds shear stress (Ganapathisubramani, Longmire \& Marusic 2003). These reports were subsequently followed by particle image velocimetry (PIV) and hot-wire observations of even larger scale motions ranging up to $40 \delta$ in streamwise direction (e.g. Kim \& Adrian 1999; Guala, Hommema \& Adrian 2006; Hutchins \& Marusic 2007; Ganapathisubramani, Clemens \& Dolling 2007b; Balakumar \& Adrian 2007), which manifest long regions of low velocity. They have been referred to as very large-scale motions (VLSM) or superstructures. Based on their signatures in onedimensional hot-wire signals, it has been conjectured that the elongated structures result from streamwise aligned hairpin packets (Kim \& Adrian 1999). It should be noted that here, and throughout the paper, the term hairpin is used to represent cane, hairpin, horseshoe and omega shaped vortices (following Adrian et al. 2000), which are believed to be variations of the same basic structure. The hairpin vortex is nowadays regarded to be more of a building block than an individual structure. For a recent review of the topic see Adrian (2007). It is noteworthy that large-scale organization of the coherent structures in the spanwise direction has not been observed and is not contemplated in these discussions.

The instantaneous flow structures in high-Reynolds-number supersonic boundary layers are currently believed to be very similar to the incompressible subsonic case. This idea is largely based on the observed similarity of the eddies at the interface of the large-scale bulges in Rayleigh scattering and oil droplet visualizations (Spina, Donovan \& Smits 1991; Smith \& Smits 1995) with further supporting evidence coming from conditional averaged flow fields obtained by hot-wire anemometry (Spina et al. 1991). It has been hypothesized that as long as the fluctuating Mach number is below 0.2 , direct compressibility effects are not important, which would be the case for free-stream Mach numbers up to 4 (Spina, Smits \& Robinson 1994). Moreover, the velocity average and fluctuation profiles collapse onto the incompressible profile when the variation of the fluid properties (i.e. temperature and density) across the boundary layer is taken into account in the scaling (Smits \& Dussauge 2006). More recently, Ganapathisubramani, Clemens \& Dolling (2006) performed PIV in a plane parallel to the wall showing very long high and low-speed regions consistent with the VLSM model (Kim \& Adrian 1999) for subsonic flow, which again suggests the presence of hairpin vortices aligned in the streamwise direction. Although the occurrence of hairpins in the supersonic boundary layer has been suggested previously on the basis of flow visualization, a direct observation in velocity measurements is lacking presently.

The complete three-dimensional structure of supersonic boundary layers is starting to become available from direct numerical simulation (Martin 2007). The returned hairpin and packets at Mach 3, $R e_{\theta}=2600$ are comparable to those found in lowReynolds-number subsonic boundary layers (Ringuette, Wu \& Martin 2008). Yet the inherent Reynolds number limitation of these simulations leaves questions regarding the structure at high Reynolds number.

The three-dimensional velocity distribution in high-speed turbulent flow has recently become accessible experimentally through the development of the tomographic PIV technique (Elsinga et al. 2006b, Elsinga 2008). This technique is a variation of standard PIV that uses volume illumination of tracer particles added to flow and records the particle images from different viewing directions simultaneously. The instantaneous particle distribution within the measurement volume is reconstructed from these images onto a discrete voxel array by a tomographic algorithm. Reconstructions are then cross-correlated to obtain the particle displacement between subsequent recordings and hence flow velocity. Having the full velocity field allows quantitative visualization of the vortical motion and computation of the three-dimensional 
conditionally averaged eddies, thereby permitting more direct interpretation of the results than in probe and planar measurements. Previous results obtained with this technique in low-speed turbulent boundary layer flows have been reported in Schröder et al. (2007) and Elsinga et al. (2007).

The present study applies the tomographic PIV technique to measure the instantaneous three-dimensional velocity distribution in a supersonic turbulent boundary layer (Mach 2, $\operatorname{Re}_{\theta}=34000$ ) in order to visualize the coherent structures and, additionally, to investigate their spatial organization in relation to the observed large-scale motions in the flow. Particular attention will go to the large-scale vortices (of size comparable to a bulge) and their organization, not only in the streamwise, but also in the spanwise direction. At this high Reynolds number the packets are expected to be long in the streamwise direction, which is beneficial to the experimental investigation as they are detected more easily. Furthermore, a large range of scales is expected. Given the similarity between the compressible and incompressible cases (as mentioned above) the present results may be relevant to subsonic boundary layers as well, especially because three-dimensional velocity information at high Reynolds numbers is scarce due to the current limitations of direct numerical simulation and experimental techniques.

Recently, the structure of the supersonic boundary layer has also received important interest in relation to shock wave turbulent boundary layer interactions with a number of studies indicating a relation between the coherent structures in the incoming boundary layer and the shock unsteadiness (e.g. Ganapathisubramani, Clemens \& Dolling 2007a, Humble et al. 2009). The present characterization of the boundary layer may contribute to this topic by providing reference data for the undisturbed turbulent flow structure.

In the remainder of the paper, the experimental set-up will be discussed $(\S 2)$ including the methods chosen for the reduction of the data aiming at vortex visualization $(\S 3)$. The analysis of the flow field is performed making use of the instantaneous snapshots $(\S 4)$ and the conditional averaged eddies inferred as from linear stochastic estimation. Furthermore, the supersonic hairpins will be compared with previous results from a low Reynolds number incompressible boundary layer $(\S 5)$. The large-scale motions are obtained from a spatial filtering $(\S 6)$ and they will be shown to exhibit a preferential alignment along the streamwise direction as well as the spanwise diagonal. This is followed by a discussion of the spatial relationship between the different vortex scales $(\$ 7)$ and a summary of the main conclusions in $\S 8$.

\section{Experimental set-up}

\subsection{Flow conditions}

The experiments were performed in the transonic-supersonic wind tunnel TST-27 at Delft University of Technology. The turbulent boundary layer over the test section sidewall was measured at a free-stream velocity $U_{e}=510 \mathrm{~m} \mathrm{~s}^{-1}$ and Mach number $M_{e}=2.1$. The flow in the boundary layer was optically accessible through the large Schlieren window in this sidewall. The boundary layer underwent natural transition far upstream in the tunnel nozzle and developed along the smooth flat tunnel wall over approximately $2 \mathrm{~m}$ before reaching the $270 \times 280 \mathrm{~mm}^{2}$ test section. At the measurement location the boundary layer thickness $\delta_{99}$ and momentum thickness $\theta$ were 20 and $1.2 \mathrm{~mm}$ respectively (for more detail on the boundary layer properties see table 1), as inferred from planar PIV measurements by Humble et al. (2006) and Humble, Scarano \& van Oudheusden (2007). The density variation necessary 


$\begin{array}{lc}U_{e} & 510 \mathrm{~m} \mathrm{~s}^{-1} \\ M_{e} & 2.1 \\ \delta_{99} & 20 \mathrm{~mm} \\ \delta^{*} & 3.7 \mathrm{~mm} \\ \theta & 1.2 \mathrm{~mm} \\ H=\delta^{*} / \theta & 3.1 \\ u_{\tau} & 19.5 \mathrm{~m} \mathrm{~s}^{-1} \\ u_{\tau} / v_{w} & 354 \mathrm{~mm}^{-1} \\ \delta^{+} & 7080 \\ c_{f} & 1.65 \times 10^{-3} \\ \operatorname{Re}_{\theta} & 34000\end{array}$

TABLE 1. Supersonic turbulent boundary layer properties (after Humble et al. 2006).

to calculate the momentum thickness was deduced from the measured velocity distribution using the adiabatic Crocco-Busemann relation with constant pressure and recovery factor $(r=0.89)$ across the boundary layer.

The definition of the relevant Reynolds number is not trivial in the case of supersonic boundary layer flow, because of the significant temperature variations over the boundary layer height (Smits \& Dussauge 2006). Perhaps the most commonly adopted is $\operatorname{Re}_{\theta}=\rho_{e} U_{e} \theta / \mu_{e}$, which is based on the free-stream fluid properties. Alternatively, the Reynolds number can be based on the shear stress at the wall resulting in $\operatorname{Re}_{\delta 2}=\rho_{e} U_{e} \theta / \mu_{w}$, where the subscripts $e$ and $w$ denote free stream and wall conditions respectively. It is still unclear which of the two should be used for comparison with subsonic boundary layers. Therefore, both are given here: $R e_{\theta}=34000$ and $R e_{\delta 2}=20000$.

\subsection{Tomographic PIV}

For the PIV measurements the flow was seeded with $240 \mathrm{~nm} \mathrm{TiO}_{2}$ particles to a concentration of approximately 3 particles $\mathrm{mm}^{-3}$ corresponding to a particle image density of 0.05 particles pixel $^{-1}$, which was sufficiently low to yield an accurate particle reconstruction (Elsinga, van Oudheusden \& Scarano 2006a, Elsinga 2008). The frequency response of these tracers was established to be $420 \mathrm{kHz}$ corresponding to a relaxation time of $2.4 \mu \mathrm{s}$ (Schrijer \& Scarano 2007). The inability to follow higher flow accelerations was not a concern here, because the measured velocity in PIV already represented an average over the time between exposures, which was close to the relaxation time in the present experiment. The particles were illuminated by a Spectra-Physics $400 \mathrm{~mJ}$ double pulse Nd:Yag laser in a light 'sheet' that was oriented parallel to the wall and was expanded to a $6.5 \mathrm{~mm}$ thickness in wall-normal direction. The sheet formation optics were contained in the light probe located downstream of the test section (figure 1). Furthermore, a slit was added to the light path to create a sharp top-hat-like light intensity profile resulting in a well-defined illumination volume. Four high-resolution CCD cameras (LaVision Imager Pro X, $2048 \times 2048$ pixels, 14 bit) within a solid angle of approximately $30 \times 30$ square degrees were used to record the particle images (figure 1) with an average resolution of 23 pixels $\mathrm{mm}^{-1}$. The lens $f_{\#}$ was set to 11 and 8.0 for the cameras in forward and backward scatter respectively. The time separation between subsequent exposures was $2 \mu \mathrm{s}$, which for the chosen volume discretization yielded a particle displacement of 20 voxels in the free stream. Both the light probe and camera system could be translated, permitting measurement of the velocity distribution at different heights 


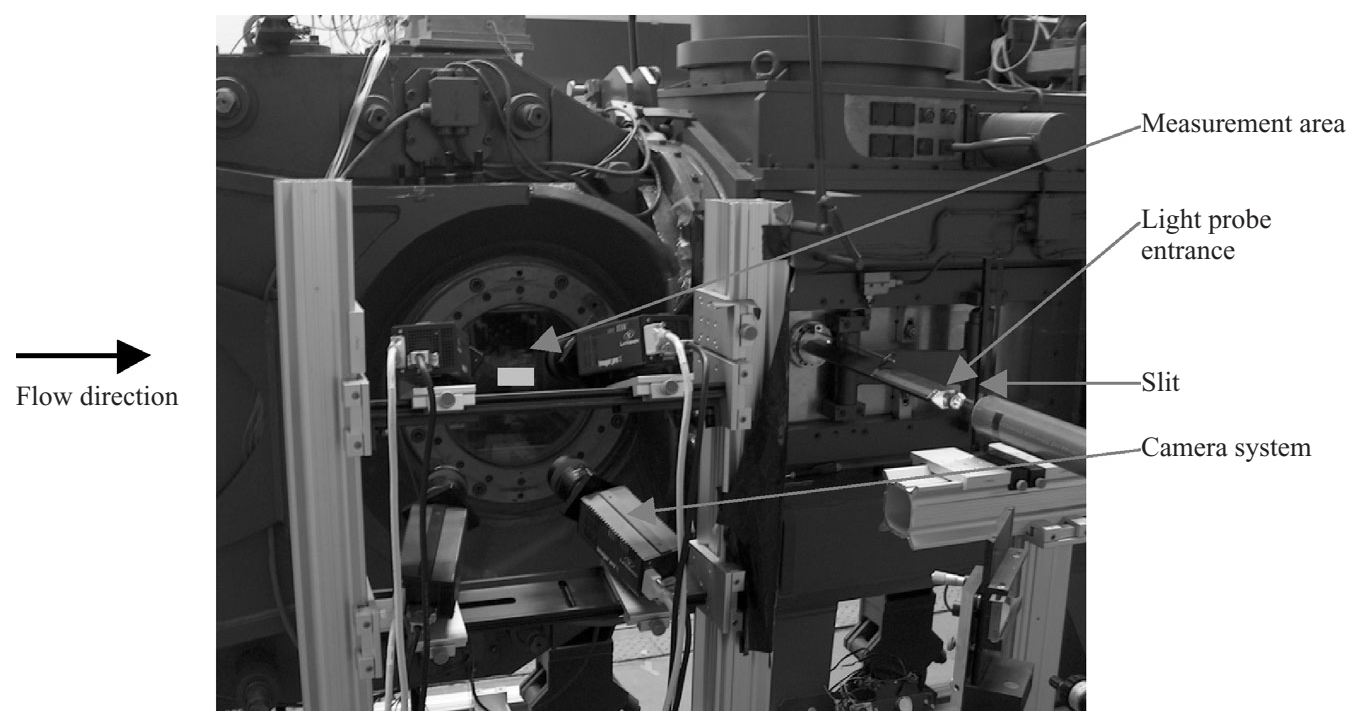

FIGURE 1. Optical arrangement at the TST-27 transonic/supersonic wind tunnel.

in the boundary layer. However, the emphasis in this paper will be on the results obtained for the configuration where the laser sheet was closest to the wall covering the region $3.0<y<9.5 \mathrm{~mm}(0.15<y / \delta<0.47)$.

The camera system was calibrated by scanning a plate with $10 \times 10$ dots through the volume in steps of $4 \mathrm{~mm}$ in depth over a total range of $8 \mathrm{~mm}$ covering the complete light sheet. In each of the three calibration planes the relation between the physical coordinates and image coordinates was described by a third-order polynomial fit. Linear interpolation was used to find the corresponding image coordinates at intermediate depth positions. Then the volume self-calibration procedure described by Wieneke (2008) was used to improve the calibration accuracy to better than 0.2 pixels for all cameras. The self-calibration correction was found to slightly increase the returned vorticity levels, but it did not affect the appearance of the flow structures.

The intensity distribution in the volume was reconstructed from the recorded images using the multiplicative algebraic reconstruction technique (MART) algorithm (Elsinga et al. 2006b) with five iterations. The reconstructed volume dimensions in streamwise $(x)$, wall-normal $(y)$ and spanwise direction $(z)$ were $70 \times 6.5 \times 35 \mathrm{~mm}^{3}$, which was discretized at $20^{3}$ voxels $\mathrm{mm}^{-3}$. The particle motion analysis was performed by cross-correlation adopting a multi-grid method including volume deformation (Scarano \& Riethmuller 2000). The measurement spatial resolution was dictated by the final interrogation box size of $40^{3}$ voxels $\left(2.0 \times 2.0 \times 2.0 \mathrm{~mm}^{3}\right)$ each including approximately 24 tracers. The cross-correlation signal-to-noise ratio, defined as the ratio of the first and second correlation peak, was approximately 2.5 . A $75 \%$ overlap factor was applied between neighbouring boxes returning a vector spacing of $0.50 \mathrm{~mm}$ in each direction (corresponding to 177 viscous wall units, $v_{w} / u_{\tau}$, based on the fluid properties at the wall) resulting in an overall measurement grid counting $142 \times 14 \times 77$ vectors. The complete dataset consisted of 480 instantaneous three-dimensional vector fields in each configuration (i.e. wall-normal position of the laser volume).

The accuracy of the present velocity measurement has been assessed in Humble et al. (2009) by comparing the root mean square (RMS) velocity profiles in the wall-normal direction with other laser Doppler anemometry (LDA) and planar PIV 
measurements. An agreement within $2.9 \mathrm{~m} \mathrm{~s}^{-1}$ was found, which corresponds to 0.15 pixel particle displacement. The latter value is consistent with the tomographic PIV uncertainty as determined in earlier comparative studies in a cylinder wake flow and an incompressible turbulent boundary layer (Elsinga 2008). Hence, the accuracy of the measurement is $0.57 \%$ of the free-stream velocity $U_{e}$, which corresponds to approximately $5 \%$ of the fluctuating velocity (taking the level of fluctuation in the boundary layer at $9 \%$ of $U_{e}$ ). For second-order moments, such as the Reynolds shear stress, the level of uncertainty would be twice, i.e. $10 \%$. From the favourable comparison of the RMS velocity profiles it can also be concluded that the energy containing turbulent motions have been resolved, even though the smallest Kolmogorov scale is clearly beyond the reach of the present measurement. It should, however, be noted here that in a high-Reynolds-number boundary layer flow, the local Kolmogorov length scale can become relatively large in the outer region; of the order of 100 wall units above approximately $y u_{\tau} / v_{w}=1000$ (e.g. Stanislas, Perret \& Foucaut 2008).

\subsection{Hot-wire anemometry}

Constant temperature hot-wire anemometry measurements were also performed to assess the large-scale low-frequency motion in the boundary layer. The hot-wire system consisted of a DISA 55M10 CTA bridge with a Dantec 55P11 single wire probe, which was made of $1.25 \mathrm{~mm}$ long, $5 \mu \mathrm{m}$ diameter tungsten wire. The equivalent wire length in viscous wall units was 443 . The overheat ratio was set to 0.88 , so that the hot wire was mainly sensitive to mass-flux fluctuations $(\rho u)^{\prime}$ and not so much to total temperature fluctuations (Smits, Hayakawa \& Muck 1983). The wire response frequency was $50 \mathrm{kHz}$, however due to a tail in the response, the measured power spectrum was affected up to $20 \mathrm{kHz}$ corresponding to a wavelength of $1.3 \delta$ using Taylor's hypothesis. The probe was translated along the wall-normal direction to measure at different $y / \delta$. The hot-wire system was calibrated using the mean voltage at different heights in the boundary layer with the corresponding mass flux obtained from the measured velocity distribution by PIV in combination with the adiabatic Crocco-Busemann relation. The main purpose for this calibration was to normalize the power spectra and not to obtain very accurate Reynolds stress levels. The accuracy of the procedure in this case was found to be limited by the accuracy of planar PIV, which typically is of the order of $1 \%$ of the mean velocity.

\section{Data reduction and display}

The flow structures of interest in this study are the low-speed zones (this terminology is used here to distinguish outer layer features from the near-wall streaks as reported, for example, by Kline et al. 1967) and the vortical structures, which often combine into hairpin packets. The former can be simply visualized by isosurfaces of the $u$ component of velocity selecting a level below the local average velocity.

Vortical motion is visualized using the $Q$ criterion (Hunt, Wray \& Moin 1988), which is based on the analysis of the second invariant $Q$ of the local velocity gradient tensor $\nabla V$. For incompressible flow this criterion can be written as:

$$
Q=\frac{1}{2}\left(|\boldsymbol{\Omega}|^{2}-|\boldsymbol{S}|^{2}\right)>0,
$$

where $\boldsymbol{S}=\left(\nabla V+(\nabla V)^{T}\right) / 2$ is the rate-of-strain tensor and $\boldsymbol{\Omega}=\left(\nabla V-(\nabla V)^{T}\right) / 2$ is the vorticity tensor. Hence $Q$ is a measure of the excess rotation rate relative to strain rate and indicates a local swirling flow topology, as shown by Chakraborty, Balachandar \& 
Adrian (2005). This kinematic interpretation allows the incompressible formulation of (3.1) to be applied in case of compressible flow as well. Moreover as previously discussed, compressibility effects are expected not to be important for the present case. A non-zero $Q$ threshold is employed to clearly identify the vortical structures separating them from the measurement noise.

$Q$ is a scalar quantity and has no direction. Therefore occasionally a twodimensional swirling strength is used to discriminate between streamwise, spanwise and wall-normal vortices. The swirling strength criterion (Zhou et al. 1999) uses the imaginary part of the complex conjugate eigenvalues $\lambda_{c i}$ of $\nabla V$, which is always positive or zero when $\nabla V$ has only real eigenvalues. Instead of the full velocity gradient tensor, the two-dimensional swirling strength uses only the velocity components and gradients in a two-dimensional plane. For instance, the spanwise swirling strength $\lambda_{c i, z}$ considers only the velocity in the $x, y$ plane and is defined as the imaginary part of the eigenvalues of $J_{u v}$ given by

$$
J_{u v}=\left[\begin{array}{ll}
\frac{\partial u}{\partial x} & \frac{\partial u}{\partial y} \\
\frac{\partial v}{\partial x} & \frac{\partial v}{\partial y}
\end{array}\right] .
$$

Similarly the steamwise swirling strength $\lambda_{c i, x}$ and wall-normal swirling strength $\lambda_{c i, y}$ are the imaginary part of the eigenvalues of $J_{v w}$ and $J_{u w}$ respectively. Finally, the two-dimensional swirling strength can be given a sign using the corresponding component of vorticity (e.g. $\lambda_{c i, z} \cdot \operatorname{sign}\left(\omega_{z}\right)$ ).

The components of the velocity gradient tensor $\nabla V$ are obtained from a secondorder regression. In this procedure the function:

$$
f_{\text {reg }}\left(r_{x}, r_{y}, r_{z}\right)=a_{0}+a_{1} r_{x}+a_{2} r_{y}+a_{3} r_{z}+a_{4} r_{x} r_{y}+a_{5} r_{x} r_{z}+a_{6} r_{y} r_{z}+a_{7} r_{x}^{2}+a_{8} r_{y}^{2}+a_{9} r_{z}^{2},
$$

is fit in the least squares sense to each component of velocity in a $5 \times 5 \times 5$ neighbourhood of a point $x^{\prime}, y^{\prime}, z^{\prime}$. The variables $r_{x}, r_{y}, r_{z}$ are distances in the $x$, $y$ and $z$ direction w.r.t. point $x^{\prime}, y^{\prime}, z^{\prime}$ and the coefficients $a_{i}$ are the fit parameters with $a_{0}$ being the filtered velocity at $x^{\prime}, y^{\prime}, z^{\prime}$ and $a_{1}, a_{2}, a_{3}$ being the local gradients in $x, y$ and $z$ respectively. The regression, also known as Savitzky-Golay filtering (Savitzky \& Golay 1964), is a linear filter, i.e. linear in $a_{i}$. Because it is not a very common filter, a comprehensive discussion of its spatial frequency response is included in the Appendix. Note that the presently applied $5 \times 5 \times 5$ kernel size is equal to the cross-correlation interrogation volume (when using $75 \%$ overlap), so that the spatial frequency response of cross-correlation and regression are similar. Consequently, the regression reduces the measurement noise without significantly affecting the measurement spatial resolution. Additionally, the regression filter will be used as a low-pass filter in $\S 6$.

For the statistical evaluation of the flow structures linear stochastic estimation (Adrian 1996; Christensen \& Adrian 2001) is used to approximate the average velocity field associated to a specified event $E$ at $x^{\prime}, y^{\prime}, z^{\prime}$. In its simplest form $E$ represents a scalar quantity obtained or derived from the measured velocity distribution, e.g. $E=u^{\prime}$ or $E=\lambda_{c i, z} \cdot \operatorname{sign}\left(\omega_{z}\right)$. The conditional average $\left\langle\hat{V}(x, y, z) \mid E\left(x^{\prime}, y^{\prime}, z^{\prime}\right)\right\rangle$ is then written as a linear function of the event $E$, where the coefficient is obtained by minimizing the mean-square error between the estimate and the conditional average. 


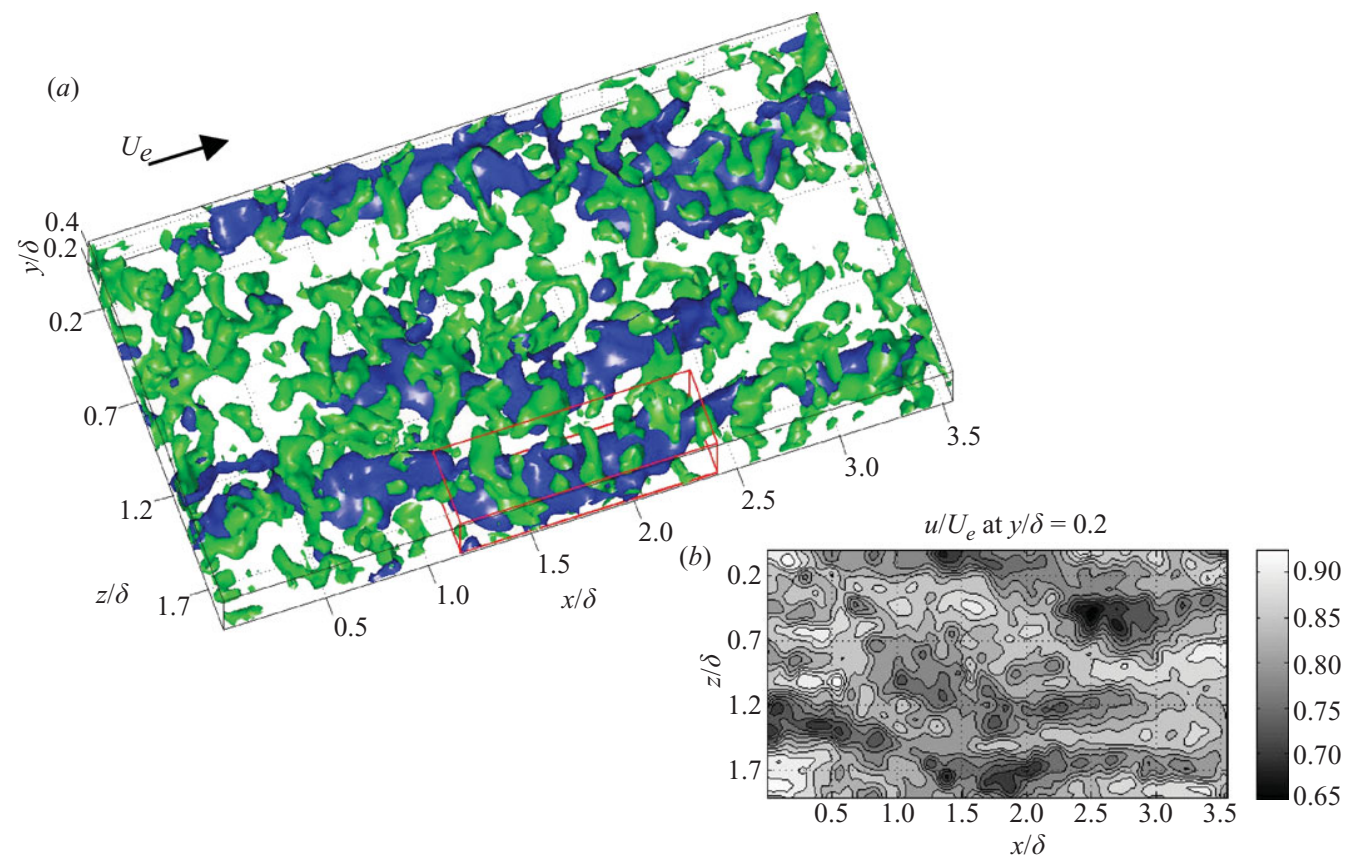

Figure 2. (a) Instantaneous vortex distribution detected by the $Q$ criterion (green) and low-speed zones (blue, $u<0.80 U_{e}$ ) for $0.15<y / \delta<0.47$. (b) A contour plot of the $u$ component of velocity at $y / \delta=0.20$.

For the fluctuating components of velocity $V^{\prime}=\left(u^{\prime}, v^{\prime}, w^{\prime}\right)$ this results in

$$
\left\langle\hat { V } ^ { \prime } \left( x^{\prime}+r_{x}, y, z^{\prime}+r_{z}\left|E\left(x^{\prime}, y^{\prime}, z^{\prime}\right)\right\rangle \approx \frac{\left\langle V^{\prime}\left(x^{\prime}+r_{x}, y, z^{\prime}+r_{z}\right) \cdot E\left(x^{\prime}, y^{\prime}, z^{\prime}\right)\right\rangle}{\left\langle E\left(x^{\prime}, y^{\prime}, z^{\prime}\right)^{2}\right\rangle} E\left(x^{\prime}, y^{\prime}, z^{\prime}\right)\right.\right. \text {. }
$$

As seen from the equation the conditional average is estimated by unconditional two-point correlations, which are much easier to compute than the conditional average itself. Furthermore, it is important to note that the estimate uses the fluctuating velocity components $V^{\prime}$ relative to the local average and that the estimate is linear, so that the character of estimate does not change with the value of $E$. Finally, if it is assumed that in a boundary layer the two-point correlations are independent of the $x$ and $z$ coordinate, then the conditional average is only a function of $r_{x}, y$ and $r_{z}$ and $\langle\ldots\rangle$ in (3.4) denotes averaging over all velocity field snapshots as well as spatial averaging in $x$ and $z$.

\section{Instantaneous flow field}

A typical example of the instantaneous flow field is presented in figure 2, where the $x$-, $y$ - and $z$-axis correspond to the streamwise, wall-normal and spanwise direction respectively. In the plot the vortical motion (green) is visualized using the $Q$ criterion (the applied threshold is $\left.10^{-9}(\partial \mathrm{u} / \partial \mathrm{y})_{w}^{2}\right)$ and the zones of low velocity (blue) are indicated by the isosurface $u / U_{e}=0.80$ corresponding to approximately $95 \%$ of the average velocity at this height in the boundary layer. Note that the visualization of the low-speed zones is not very sensitive to the applied threshold, since they are separated from the high-speed flow by regions of high shear, as for instance can be 
seen in the $u$ contour plot of figure 2. This is much like the zones of relatively uniform momentum observed by Meinhart \& Adrian (1995). The low-speed zones are several boundary layer thicknesses long, and they often extend beyond the measurement volume. Ganapathisubramani et al. (2007b) even report lengths up to $40 \delta$ at $y / \delta=0.2$ in their supersonic boundary layer visualization based on high repetition rate PIV measurements and using Taylor's hypothesis. Long low-speed zones have also been found in incompressible turbulent boundary layers and pipe flow using a single hot-film (Kim \& Adrian 1999) or a spanwise array of 10 hot wires to account for meandering of the low-speed zones (Hutchins \& Marusic 2007). The width of the low-speed zones observed in the present experiments varies between $0.25 \delta$ and $0.4 \delta$, which is consistent with earlier studies (Spina et al. 1991; Tomkins \& Adrian 2003; Ganapathisubramani et al. 2006; Ringuette et al. 2008), and the spanwise spacing between low-speed zones is typically between $0.5 \delta$ and $1.0 \delta$.

At larger distances from the wall (figure 3) the low-speed zones decrease in length, whereas their width changes very little, based on the visual inspection of the instantaneous results. These observations are consistent with the reported decreasing correlation lengths of $u^{\prime}$ in streamwise direction with distance from the wall above $y / \delta=0.16$ and the slowly increasing correlation length in spanwise direction (Spina et al. 1991; Ganapathisubramani et al. 2006). Furthermore, above $y / \delta=0.6$ the coherent structures, i.e. low-speed zones and vortices, show intermittent behaviour, meaning that some snapshots contain only a few separate vortices, while other snapshots contain distinct elongated low-speed zones that appear similar to the case presented for $0.34<y / \delta<0.67$ (figure $3 a$ ). The example given here for $0.56<y / \delta<0.89$ (figure $3 b$ ) represents the intermediate case, where the volume contains a number of short low-speed regions surround by vortical motion.

Plots of the complete measurement volume, such as in figures 2 and 3, reveal that most vortical structures are concentrated near the low-speed zones. However at this scale of presentation the character of the individual vortices cannot be appreciated properly. Therefore a detail taken from the volume of figure 2 is enlarged and shown in figure 4(a). Over the low-speed zone the heads from a series of streamwise aligned hairpin (or arch) vortices are visible, which can be considered a hairpin packet (Adrian et al. 2000). Note that the arch located at $x / \delta=1.7$ is partially contained in the lowspeed zone and may therefore appear fragmented due to the blue isosurface blocking the view. The streamwise spacing of the vortices inside this packet is approximately $0.2 \delta$. Figure $4(b, c)$ presents the corresponding velocity vector field after subtraction of a convective velocity in two perpendicular cross-sectional planes. The velocity distribution in the $x, y$ plane (figure $4 b$ ) shows swirling motion around the heads of the individual hairpins. For clarity the circles indicate the location of the vortices as given by the $Q$ criterion. It is also seen that the vortex heads convect with slightly different velocities, what will result in the interaction or merging of the vortices at a later stage. Furthermore, the vortices are of approximately the same size and do not appear to be aligned along a $12^{\circ}-20^{\circ}$ slope with the wall as in Head \& Bandyopadhyay (1981) or in the logarithmic layer vortex packets observed in Adrian et al. (2000). Near the top of the volume at $y / \delta=0.45$ the velocity direction and magnitude is very irregular, which may indicate the presence of (vortical) flow structures just above this packet. Finally, the distribution in the $x, z$ plane (bottom diagram) shows swirling motion around the necks of the hairpins. Most of the hairpins are asymmetric (i.e. a cane type vortex) showing just a single neck on one side of the low-speed region. 

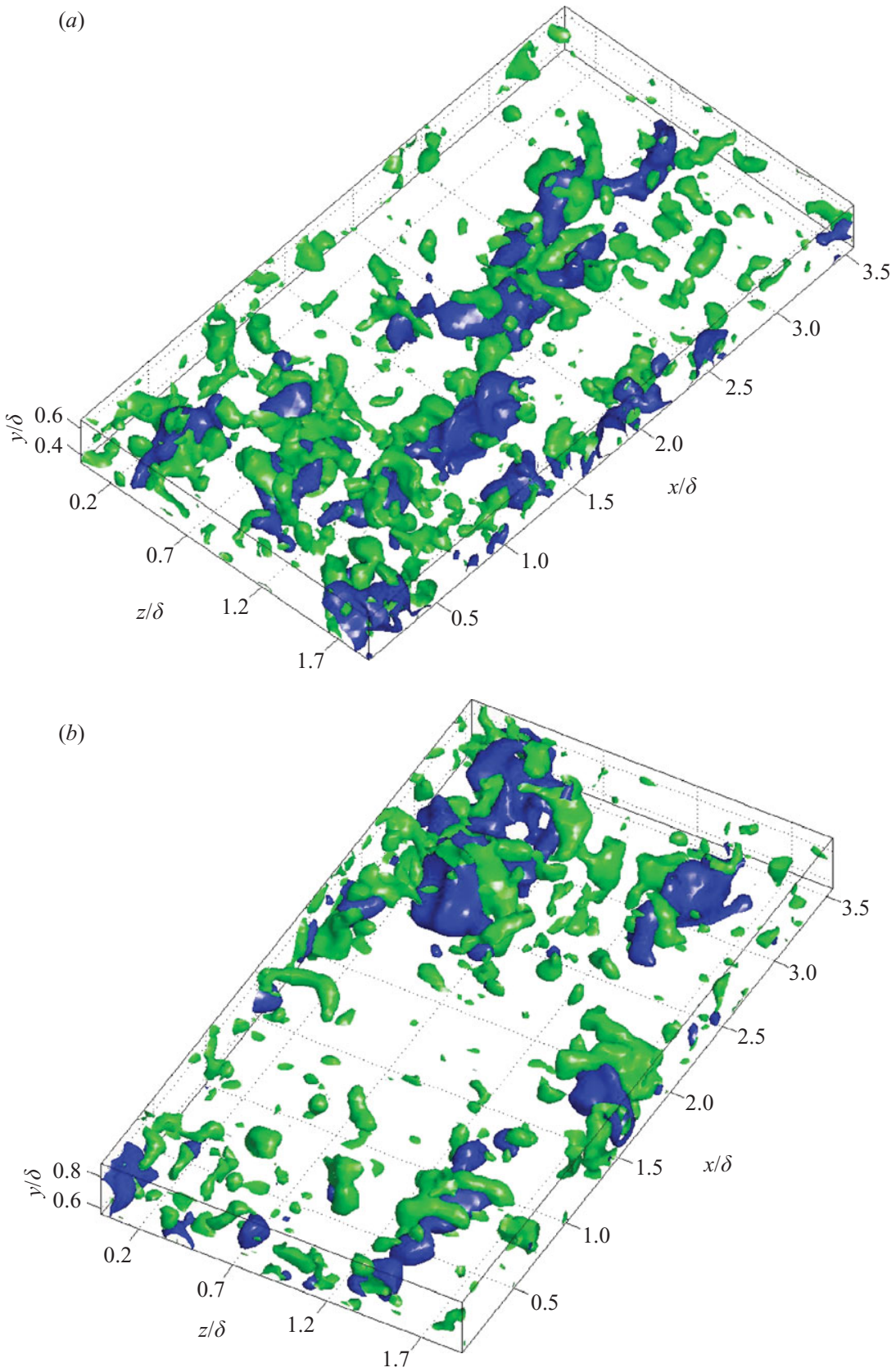

FIGURE 3. Instantaneous vortex distribution detected by the $Q$ criterion (green) and low-speed zones (blue) for $0.34<y / \delta<0.67\left((a)\right.$ blue $\left.u<0.89 U_{e}\right)$ and $0.56<y / \delta<0.89$ ((b) blue $\left.u<0.94 U_{e}\right)$.

\section{Hairpin vortices}

In order to further examine the individual vortex structures, two typical examples of individual hairpin vortex structures are shown in figures $5(a)$ and $5(b)$. The first one is the red coloured vortex in the packet of figure 4. It exhibits an asymmetrical 

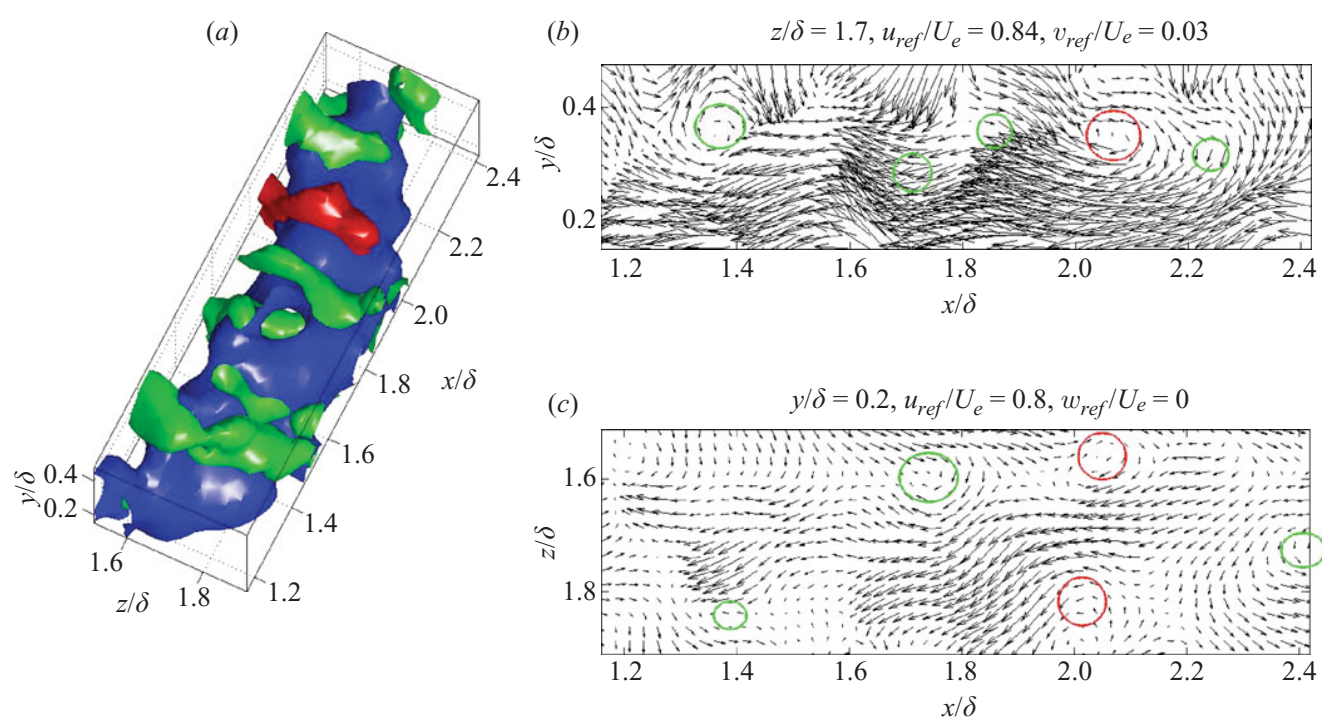

Figure 4. (a) Arch vortices around a low-speed zone in the sub-volume indicated in figure 2 and $(b, c)$ corresponding vector plots in the $x, y$ and $x, z$ cross-sections. A detailed view of the vortex indicated in red is presented in figure 5. Other colours as in figure 2.
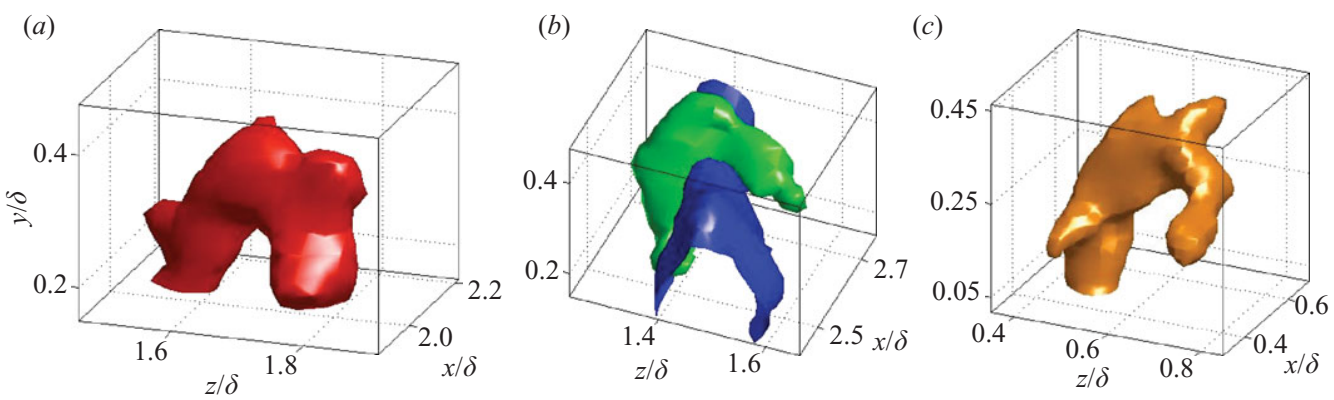

FiguRE 5. Individual vortices. (a) Arch extracted from the volume of figure 4. (b) Example of a cane vortex and low-speed zone (colour-coding as in figure 2). (c) Cane vortex observed in a low-speed incompressible boundary layer (Elsinga et al. 2007).

arch shape, and its height and width are $0.33 \delta$ and $0.2 \delta$ respectively. The irregularities on the surface of this hairpin, i.e. the blobs attached to the arch, may be either due to measurement noise or ascribed to smaller scale motions near the arch that have not been fully resolved and it is well known that a local vortex detection scheme like the $Q$ criterion cannot distinguish between individual vortices in close proximity.

The second vortex (green, figure $5 b$ ) is of a cane type, and it is also found near an extended low-speed zone (blue). It is slightly higher $(0.4 \delta)$ compared to the previous example, but has approximately the same width $(0.2 \delta)$. The width of both vortices corresponds to the width of the low-speed zones, as mentioned above in $\S 4$.

Additional statistical evidence for the existence of hairpin vortices at the present Reynolds and Mach number is given by the conditional eddy obtained by linear stochastic estimation, which reveals an omega shaped hairpin (green, figure $6 a, b$ ). The event used for the conditional average is the spanwise swirling strength $\lambda_{c i, z} \cdot \operatorname{sign}\left(\omega_{z}\right)$ at $y / \delta=0.35$ considering only negative values. As seen from figure 6 this event 
(a)
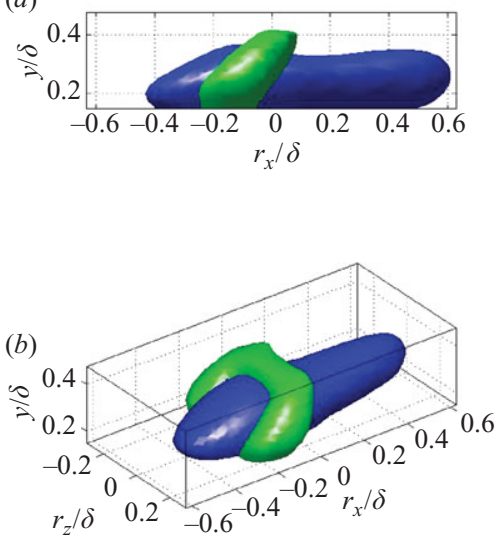

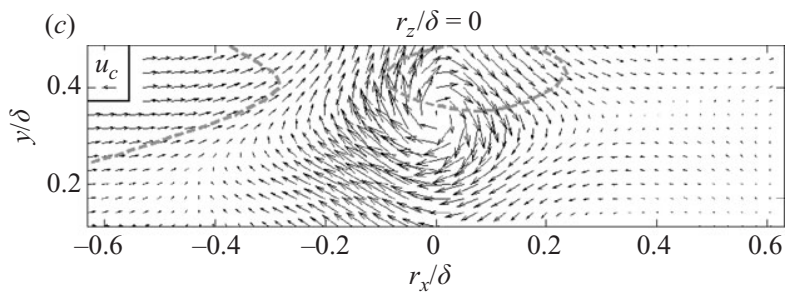

$(d)$

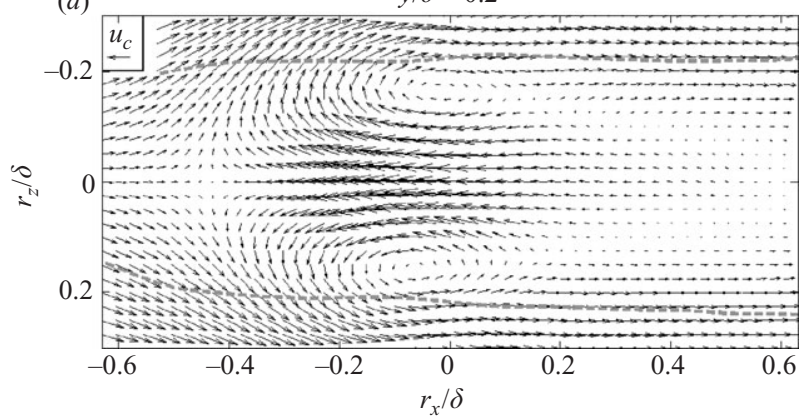

FIGURE 6. Conditional eddy $(a, b)$ given a negative spanwise swirling event at $y / \delta=0.35$ visualized using the $Q$ vortex detection criterion (green) and low velocity region (blue, $\hat{u}^{\prime}<u_{c}^{\prime}$ ) with corresponding velocity vector plot in the $x, y$ plane at $r_{z}=0$ and $x, z$ cross-sections at $y / \delta=0.2(c, d)$. The velocity vectors are relative to the eddy convective velocity $u_{c}^{\prime}$, at $y / \delta=0.35$ indicated in the upper left corner of each plot. The dashed lines indicate $\hat{u}^{\prime}=0$.

corresponds to a hairpin head at the specified location. The neck of the conditional eddy is at a $45^{\circ}$ angle with the wall, as expected, and the eddy width is about $0.25 \delta$ (based on the distance between the neck centres as detected by $Q$ ) in agreement with the observed width of the instantaneous hairpins (figure 5), the low-speed zones (figure 2) and the width versus height relation for the conditional eddies found in Tomkins \& Adrian (2003). Underneath the eddy a $1.0 \delta$ long region is visible where the velocity is smaller than the convective velocity of the conditional eddy $\left(\hat{u}^{\prime}<u_{c}^{\prime}\right.$, blue). The convective velocity $u_{c}^{\prime}$ is taken as the velocity returned by the linear stochastic estimation procedure at the event location $\left(r_{x} / \delta=r_{z} / \delta=0, y / \delta=0.35\right)$. Relative to the ensemble average velocity $u_{c}^{\prime}$ has a negative value, indicating the conditional eddy advects at a speed smaller than the local average velocity. In contrast, Adrian et al. (2000) found the heads of instantaneous hairpins convected on average at the mean velocity corresponding to the location of the centre of the head. The interface between the low and high-speed fluid upstream of the eddy is inclined at approximately $25^{\circ}$ with the wall.

Figure $6(c, d)$ presents the corresponding fluctuating velocity vectors relative to $u_{c}^{\prime}$ in the $x, y$ symmetry plane at $r_{z}=0$ and the $x, z$ plane parallel to the wall at $y / \delta=0.2$. The convective velocity is indicated in the upper left corner of each plot and has been subtracted from the velocity distribution to highlight the swirling motion around the head and necks of the eddy. The vectors in the $x, y$ plane also show a shear layer upstream of the hairpin and an ejection event $\left(u^{\prime}<0, v^{\prime}>0\right)$ between the legs of the hairpin, as expected. Furthermore, the vectors in the $x, z$ plane show a stagnation point in the symmetry plane at $r_{x} / \delta=-0.42$ and upstream fluid flowing around the eddy. Although not readily apparent from the vectors in figure 6, because of the subtraction of $u_{c}^{\prime}$ the spanwise swirling event does correlate with elongated regions of low velocity with respect to the local average value. This 
can be seen from the contour line $\hat{u}^{\prime}=0$ (dashed lines), which reveal a low-speed region approximately $0.4 \delta$ wide and extending at least $1.2 \delta$ in positive and negative streamwise direction. The slope of the contour in the $x, y$ symmetry plane upstream of the eddy $\left(r_{x} / \delta<0.3\right)$ is approximately $18^{\circ}$ somewhat larger than the $14^{\circ}$ reported in Christensen \& Adrian (2001). The discontinuity in the $\hat{u}^{\prime}=0$ contour upstream of the eddy in the symmetry plane is unexpected and is believed to be related to minor inaccuracies in the measurement to which contours are sensitive, rather than to a flow feature. The fact that the conditional eddy is contained in a low-speed environment that is clearly wider and much longer than what can be expected for a single hairpin or perhaps even a small hairpin packet, suggests the presence of large-scale flow structures. This will be explored further in $\S 6$.

Conditional eddies have also been computed for negative spanwise swirl events at different distances from the wall. It was found that the shape of the conditional eddy did not change appreciably in this part of the boundary layer (well above the logarithmic layer). The width of the averaged hairpin, however, increased slowly with its height consistent with Townsend's attached eddy hypothesis and the trend in the spanwise correlation length of $u^{\prime}$. As an example, for a hairpin head located at $y / \delta=0.75$ the returned eddy width increased to $0.38 \delta$. Even though these appear to be attached eddies, in the sense that their size scales with their distance to the wall, they may still be detached in the sense that they do not extend all the way down to the wall. This point must be left open here, as the velocity has been measured only above $y / \delta=0.15$.

For comparison, a hairpin structure returned by tomographic PIV in an incompressible turbulent boundary layer at $R e_{\theta}=1900$ (Elsinga et al. 2007; Elsinga 2008) is shown in figure 5(c). The shape is very similar to the hairpins found in the supersonic boundary layer, as is its size when scaled with the boundary layer thickness (height $0.35 \delta$ and width $0.25 \delta$ ). Similarity of the flow pattern is also evident from the conditional eddy as shown by the velocity vectors in figures 6 and 7 for the supersonic and incompressible case respectively. This seems even more remarkable when considering that the spatial resolution based on the cross-correlation volume in both experiments is very different: 708 versus 56 viscous wall units or $0.10 \delta$ versus $0.067 \delta$. However, in both experiments the energy containing motions have been resolved, as evident from the RMS profiles of fluctuating velocity, which compare to within a few percent with other, high spatial resolution experimental datasets. The present results reconfirm that the asymmetric hairpin structure is a universal feature of the energy containing motions in a turbulent boundary layer, also in supersonic flow conditions. As mentioned before, the width of these hairpins appears to be closely related to the width of the low-speed zones. Evidence in incompressible boundary layers suggests that the latter scales on $\delta$ over a range of Reynolds numbers (Hutchins \& Marusic 2007), which provides some further indirect support for an outer scaling of the hairpins, at least above $y / \delta=0.2$.

\section{Large-scale structures}

\subsection{Pre-multiplied power spectra}

The pre-multiplied power spectrum is used to obtain the characteristic size of the large flow structures. In this plot the power spectrum for each velocity component $\Phi_{u u}(k), \Phi_{v v}(k)$ and $\Phi_{w w}(k)$ is multiplied by the wavenumber $k$ so that the area under the log-linear graphs of $k \Phi$ versus $k$ corresponds to the kinetic energy for each velocity component. Hence the pre-multiplied power spectrum can be used to 

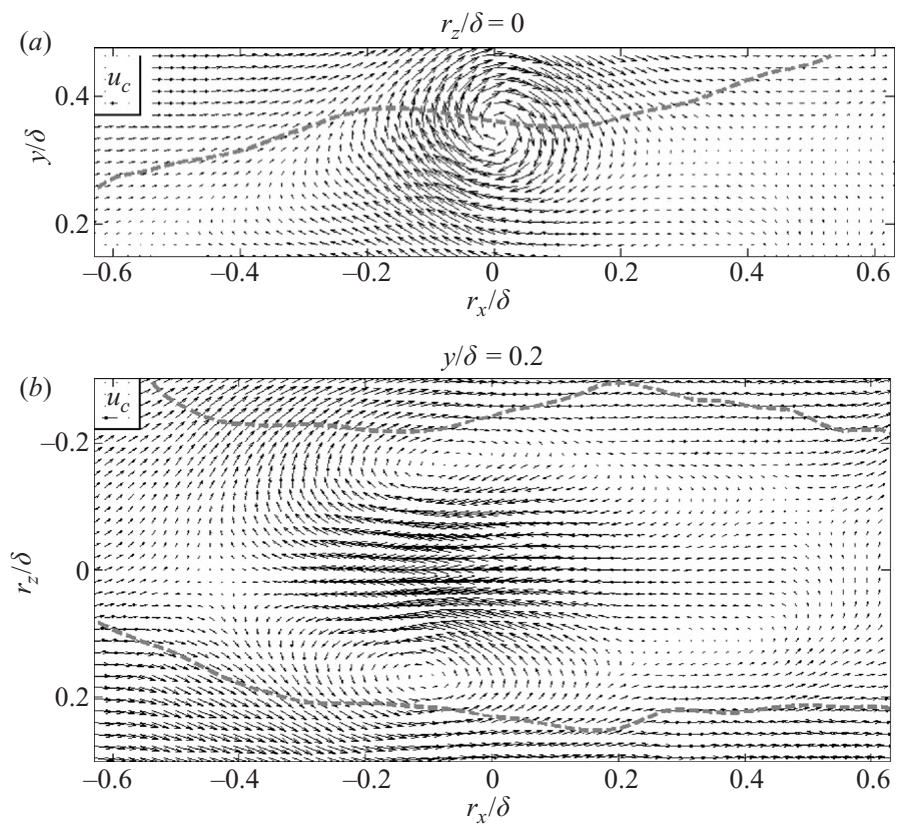

FIGURE 7. Conditional eddy in a low-speed incompressible boundary layer for comparison with the supersonic conditional eddy in figure 6.
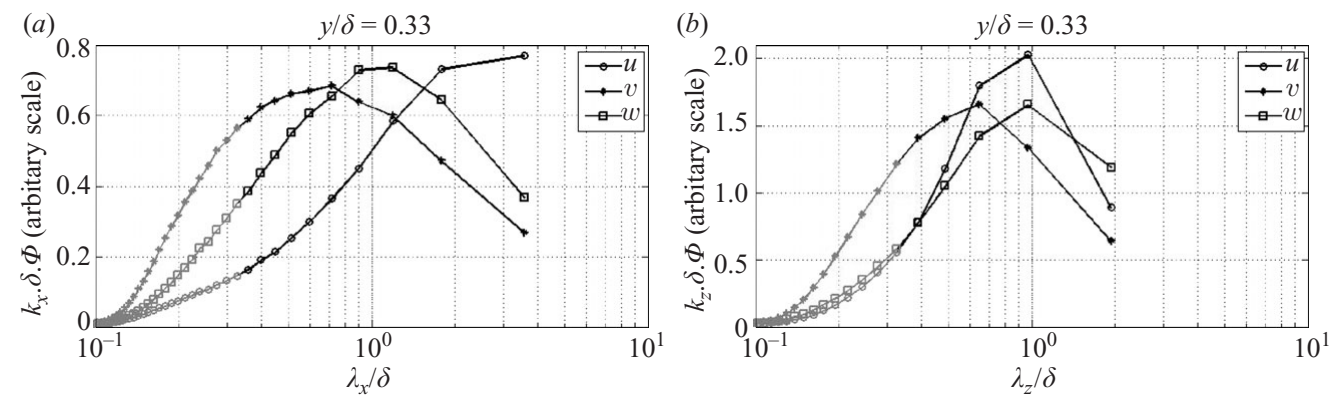

FIGURE 8. Velocity pre-multiplied power spectra as a function of streamwise wavelength (a) and spanwise wavelength $(b)$.

show the contributions of the different wavenumbers $k$, or alternatively wavelengths $\lambda=2 \pi / k$, to the kinetic energy. Figure 8 presents the pre-multiplied spectra for streamwise wavelengths $\lambda_{x}$ and spanwise wavelengths $\lambda_{z}$ in the present boundary layer at $y / \delta=0.33$. In this figure the area under the plot is normalized, so only the relative contributions of the different wavelengths are shown. Furthermore, wavelengths below three times the cross-correlation volume size are strongly affected by amplitude modulation rendering the data unreliable for these wavelengths. They are, however, included in the plot (in grey) for completeness.

In the spanwise spectra (figure $8 b$ ) a peak contribution is found between $\lambda_{z} / \delta=0.7$ and 1.0 for all velocity components, which is the wavelength associated to spanwise distribution of the low- and high-speed zones and their related (vortex) flow structures. In contrast the streamwise spectra (figure 8a) show peaks at clearly different wavelengths for each velocity component. Most noticeably the $u$ component does 


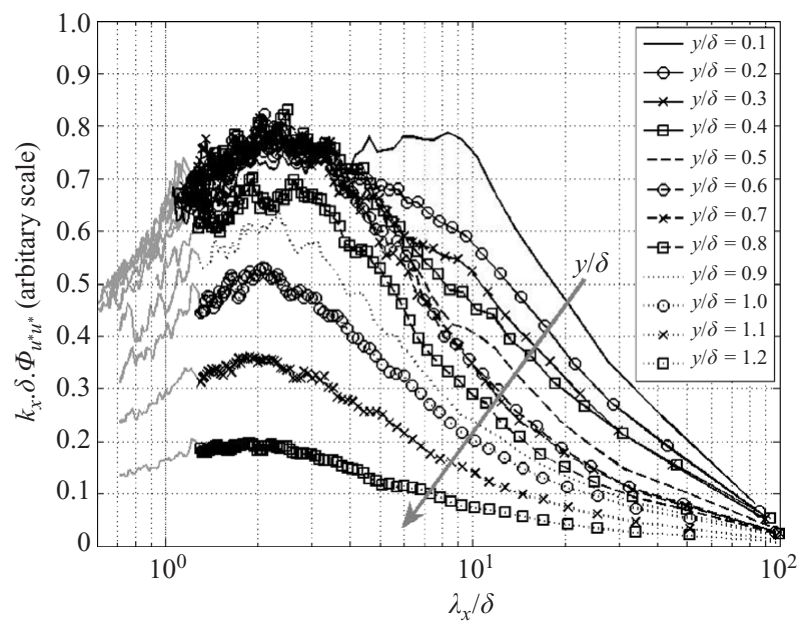

Figure 9. Pre-multiplied power spectra obtained by hot-wire anemometry across the boundary layer.

not peak, but continues to increase beyond $\lambda_{x} / \delta=3$, which must be attributed to long streamwise lengths of the low- and high-speed zones extending beyond the measurement volume. The $v$ component of velocity returns a broad peak ranging from approximately $\lambda_{x} / \delta=0.4$ to 0.9 . It is believed that the broad peak results from a combination of smaller wavelengths, say $\lambda_{x} / \delta=0.4$, related to the hairpins shown in $\S 5$, and larger scales of the order $\lambda_{x} / \delta=1$. Finally, the streamwise spectrum for the $w$ component has a more distinct maximum at $\lambda_{x} / \delta=1.2$, which can be regarded as a typical size for a turbulent bulge.

To complete the pre-multiplied power spectrum for the $u$ component of velocity towards the larger streamwise length scales, hot-wire anemometry is used. The measured frequency information is converted to length scales by applying Taylor's hypothesis. Moreover, the effect of density variations across the boundary layer on the measured momentum fluctuations $(\rho u)^{\prime}$ is relieved by considering $u^{*}$, which is defined as

$$
u^{*}=\frac{(\rho u)^{\prime}}{\bar{\rho}},
$$

where $\bar{\rho}$ is the local average density obtained from the adiabatic Crocco-Busemann relation.

The resulting pre-multiplied power spectra are shown in figure 9 for several wall-normal coordinates $y / \delta$. It is found that above $y / \delta=0.2$ all spectra peak around $\lambda_{x} / \delta=2$, which length scale is accessible within the present Tomographic PIV measurement volume. Furthermore the energy contained in this length scale is approximately constant up to $y / \delta=0.7$, after which it decreases with distance from the wall. For $y / \delta=0.1$ a peak is observed near $\lambda_{x} / \delta=9$, which indicates the presence of VLSM at that height in the boundary layer (Kim \& Adrian 1999). The energy contained in the very large scales decreases with distance from the wall, so that they are visible only as a small bump in the power spectra up to $y / \delta=0.5$. This behaviour of the peak is in agreement with the incompressible boundary layer results of Balakumar \& Adrian (2007). Note that the actual length is believed to be underestimated by the hot-wire data due to meandering of these very large-scale structures (Hutchins \& Marusic 2007; Ganapathisubramani et al. 2007b). Furthermore, below $\lambda_{x} / \delta=1.3$ the 
pre-multiplied power spectra are slightly affected by the response of the hot wire. The data is, however, included to illustrate the trend.

From the power spectra it is anticipated that the largest possible vortical structures are approximately $1.2 \delta$ long. This may be inferred by considering that a swirling motion is always composed out of at least two different fluctuating velocity components (e.g. $\lambda_{c i, x}$ is a function of $v$ and $w$ ), which then need to have a similar energy content at the same wavelength for this motion to possibly be coherent in space. Because only the spectrum for $u$ is seen to increase beyond $\lambda_{x} / \delta=1.2$ while the other two drop off, a swirling motion cannot be coherent over much larger length scales. In the same way the largest spanwise length scale is estimated at $1.0 \delta$. The power spectra, however, do not confirm that these motions do indeed exist at that scale nor do they reveal what the actual shape of these vortices should be. For that, a spatial low-pass filtering of the velocity fields is required, which suppresses the small-scale patterns emphasized in visualizations of differential quantities such as vorticity and swirling strength.

\subsection{Filtered velocity fields}

The second-order regression of (3.3) is used to low-pass filter each velocity component with the purpose of bringing forward the topology of the large-scale flow structures. The second-order polynomial is fit to the velocity in a volume measuring $0.78 \delta \times 0.18 \delta \times 0.28 \delta$ in $x, y$ and $z$ direction. The corresponding filter wavelength for three-dimensional velocity variations can be estimated as twice the fitted volume: $1.5 \delta \times 0.35 \delta \times 0.55 \delta$ (see Appendix). The filter wavelength in the streamwise direction coincides with the expected length of the largest vortical structures as argued above. The filter size in the spanwise direction is chosen to match the width of the low-speed zones. Finally, the filter wavelength in the wall-normal direction is necessarily small to allow variation over the height of the measurement volume.

In the measurement volume closest to the wall, the low-pass filtered velocity fields return predominately streamwise and wall-normal vortices, which are visualized in figure 10 by the two-dimensional swirling strengths $\lambda_{c i, x}$ and $\lambda_{c i, y}$ respectively. Furthermore, it is seen that both types of vortices occur next to low-speed zones. The strength of the vortices based on their peak $Q$ value is about $1 / 4$ of the hairpins presented in $\S 5$. Streamwise vortices are typically inclined at $5^{\circ}$ with the wall and are $0.8 \delta-1.0 \delta$ long. Wall-normal vortices often occur in counter rotating pairs on opposite sides of the low-speed zones, which is the typical hairpin signature in the $x, z$ plane. Moreover, streamwise vortices have been frequently observed to terminate in the wall-normal vortices suggesting that they may be considered as legs to cane or hairpin structures. It is important to note that applying the $Q$ criterion instead of the two-dimensional swirling strength returns the same vortex structures but merged, which would have made the interpretation of the results more difficult.

The average properties of the observed wall-normal vortices are investigated further by calculating the conditional eddy based on the event $\lambda_{c i, y} \cdot \operatorname{sign}\left(\omega_{y}\right)$ (wall-normal swirling strength) in the low-pass filtered velocity fields at $y / \delta=0.33$, considering only positive values. The result is presented in figure 11 showing two counter-rotating vortices inclined at $45^{\circ}$ with the wall (magenta) and with a low-speed zone in between, which is shown by the isosurface of the fluctuating streamwise component of velocity $\hat{u}^{\prime}=0$ (blue). Together the vortices and low velocity again constitute a hairpin vortex signature, where the vortices visualized in figure 11 are part of a neck on either side of the hairpin head. As will be shown later, the hairpins are often skewed, which results in the reduced swirling strength in the second hairpin neck $\left(r_{z} / \delta=-0.4\right)$ due to 


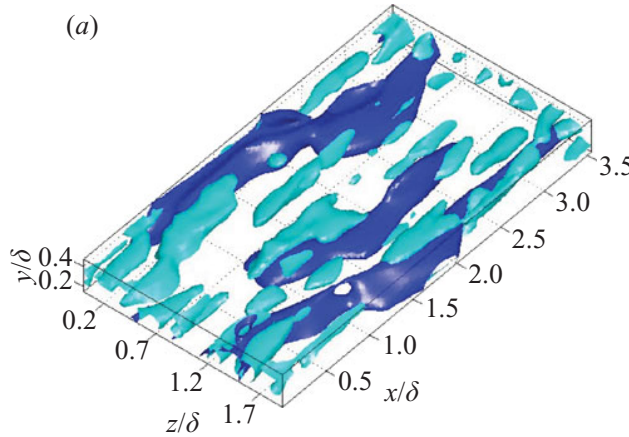

(b)

Figure 10. Streamwise (cyan $(a)$ ), wall-normal swirling motion (magenta $(b)$ ) and low-speed zones (blue) in the low-pass filtered velocity field of figure $2(0.15<y / \delta<0.47)$.

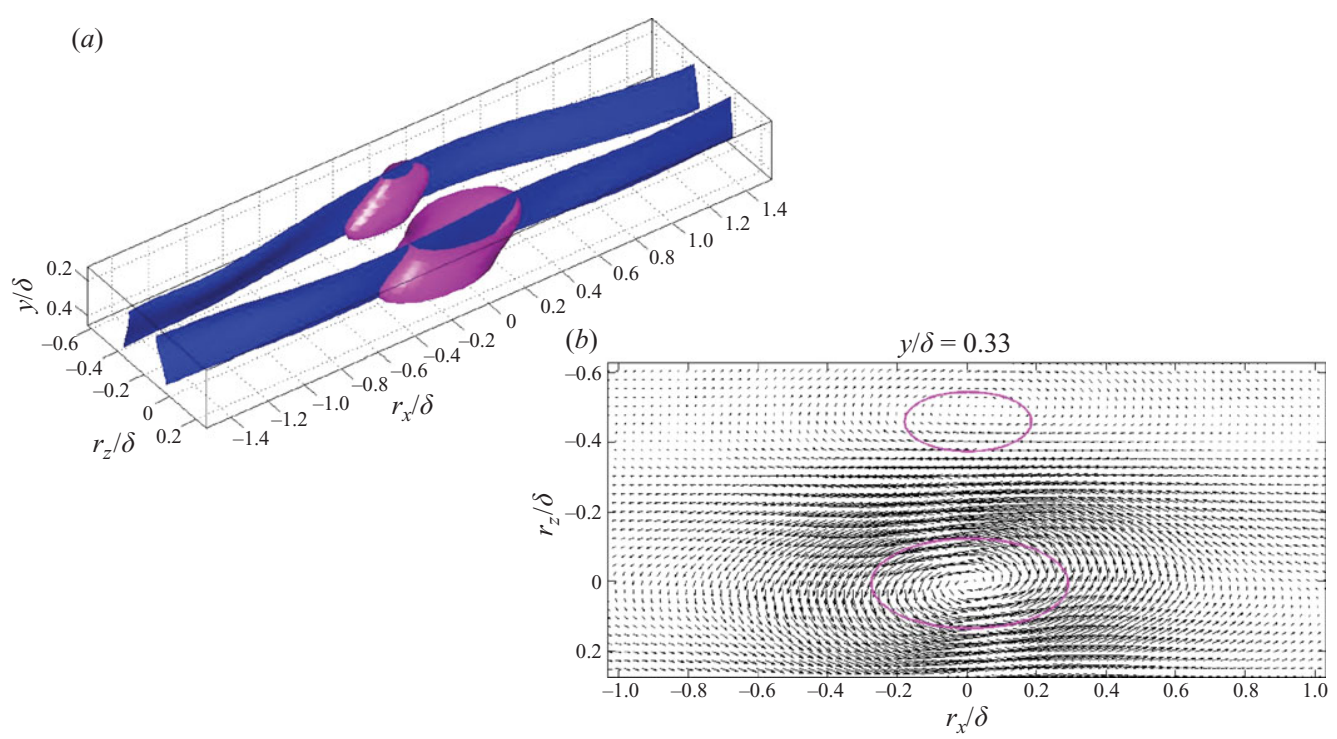

Figure 11. Conditional eddy $(a)$ given a positive wall-normal swirling event in the low-pass filtered data at $y / \delta=0.33$ visualized using the $Q$ vortex detection criterion (magneta) and low-speed zones (blue, $\hat{u}^{\prime}<0$ ) with corresponding vector plot in the $x, z$ cross-section $(b)$. The velocity vectors are relative to the local average velocity.

averaging. It should be stressed that no convective velocity has been subtracted from the vector field here, because the large-scale hairpins are advected at approximately the local average velocity. The conditional average shows furthermore that the largescale hairpin is related to very long zones of relative low velocity approximately $0.4 \delta$ wide, which is nearly identical to the large-scale low-speed region encountered before in the conditional average of the smaller scale hairpins in figure 6. This may suggest that these long low-speed zones are actually formed by the large-scale vortices and that the smaller scale hairpins are in turn more local features contained inside the low-speed zones associated to the large-scale vortices (see also §7).

More evidence for the existence of large-scale hairpins is given by the hairpin heads commonly observed in the volumes covering the region $0.56<y / \delta<0.89$. Two examples showing vortical motion (magenta) and low-speed zones (blue) in the lowpass filtered velocity fields at that height are presented in figure 12 . In the left volume 

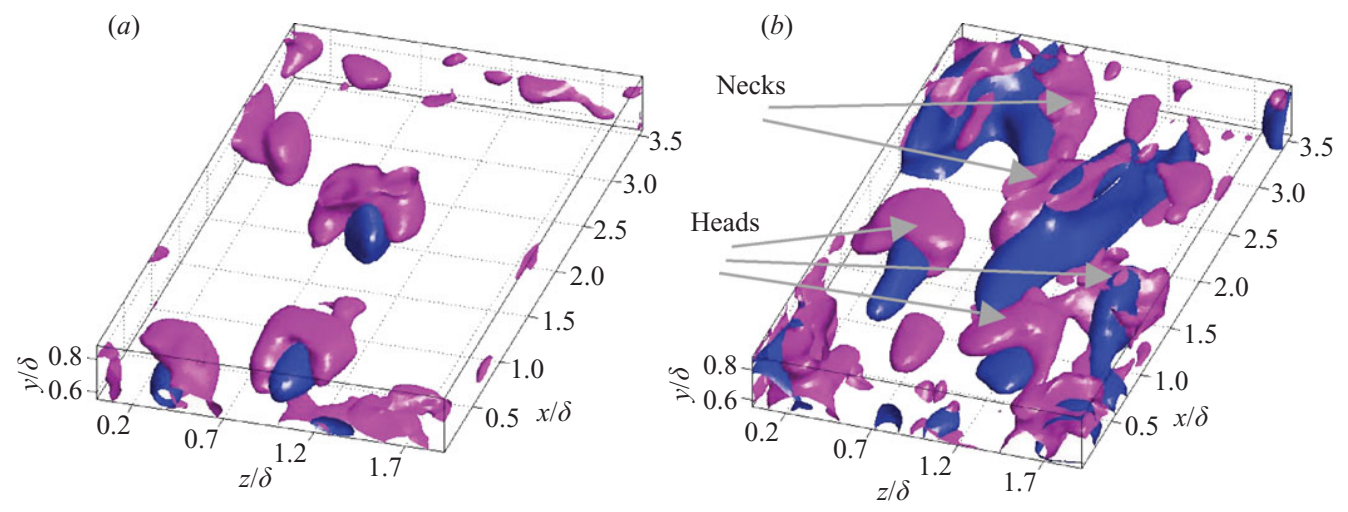

FIGURE 12. Hairpin heads observed in the low-pass filtered velocity fields above $y / \delta=0.6$ visualized using the $Q$ criterion (magenta) and low-speed zones (blue, $u<0.95 \cdot U_{e}$ ).

isolated arches and canes are seen with a short low-speed zone between the necks, as expected. The second volume (figure $12 b$ ) reveals the streamwise arrangement of an arch vortex and a neck around an elongated low-speed zone. The neck may belong to an even taller hairpin vortex structure. Taking the energy around $\lambda_{x} / \delta=2$ in the pre-multiplied power spectrum $k_{x} \Phi_{u u}$ (figure 9) as directly representative of the large-scale hairpins, the decreasing energy peak value for $y / \delta>0.7$ may indicate that the large-scale hairpins are approximately $0.7 \delta$ tall, which agrees with the present findings that very few heads are returned in the low-pass filtered volumes closer to the wall. Furthermore it is noted that at the upper half of the boundary layer large-scale streamwise vortices have rarely been observed.

The large-scale hairpins described here are not unlike the turbulent bulges in terms of their dimensions and the notion of large-scale flow rotation (Blackwelder \& Kovasznay 1972; Falco 1977). The hairpin inclination together with its arch shape also explains the strong streamwise velocity gradient commonly found upstream of the bulge compared to the weaker gradient downstream. Furthermore, the results are consistent with the $45^{\circ}$ structure angle as deduced from dual hot-wire measurements with a large wall-normal spacing in a supersonic boundary layer (Spina et al. 1991) and falls within the $30^{\circ}-70^{\circ}$ range for the density structures visualized by Schlieren techniques (Smith \& Smits 1995; Garg \& Settles 1998).

\subsection{Very large-scale diagonal flow pattern}

In the instantaneous low-pass filtered velocity field alignment of the large-scale hairpins is frequently observed along diagonal lines. One of the clearest examples of diagonal alignment is presented in figure $13(b)$ showing velocity vectors and wallnormal swirl $\lambda_{c i, y}$ contours in the plane parallel to the wall at $y / \delta=0.33$. The hairpin signatures have been indicated in the plot by circles and the dashed lines indicate the diagonals along which the hairpins appear to be aligned. Figure 13(b) also shows that the hairpin vortices are often skewed or appear one-sided, i.e. of cane type. For comparison the original (unfiltered) velocity distribution in the same plane is presented in figure 13(a). Between the filtered and unfiltered plots little difference is observed in the low-speed zones. However, the difference in vortical structures is profound, appearing almost random in the unfiltered data, but clearly more organized after filtering. The applied filter has a relatively small local support (i.e. considers vectors only within the $0.78 \delta \times 0.18 \delta \times 0.28 \delta$ size kernel), which makes it unlikely that the observed organized pattern is introduced by the filter. 

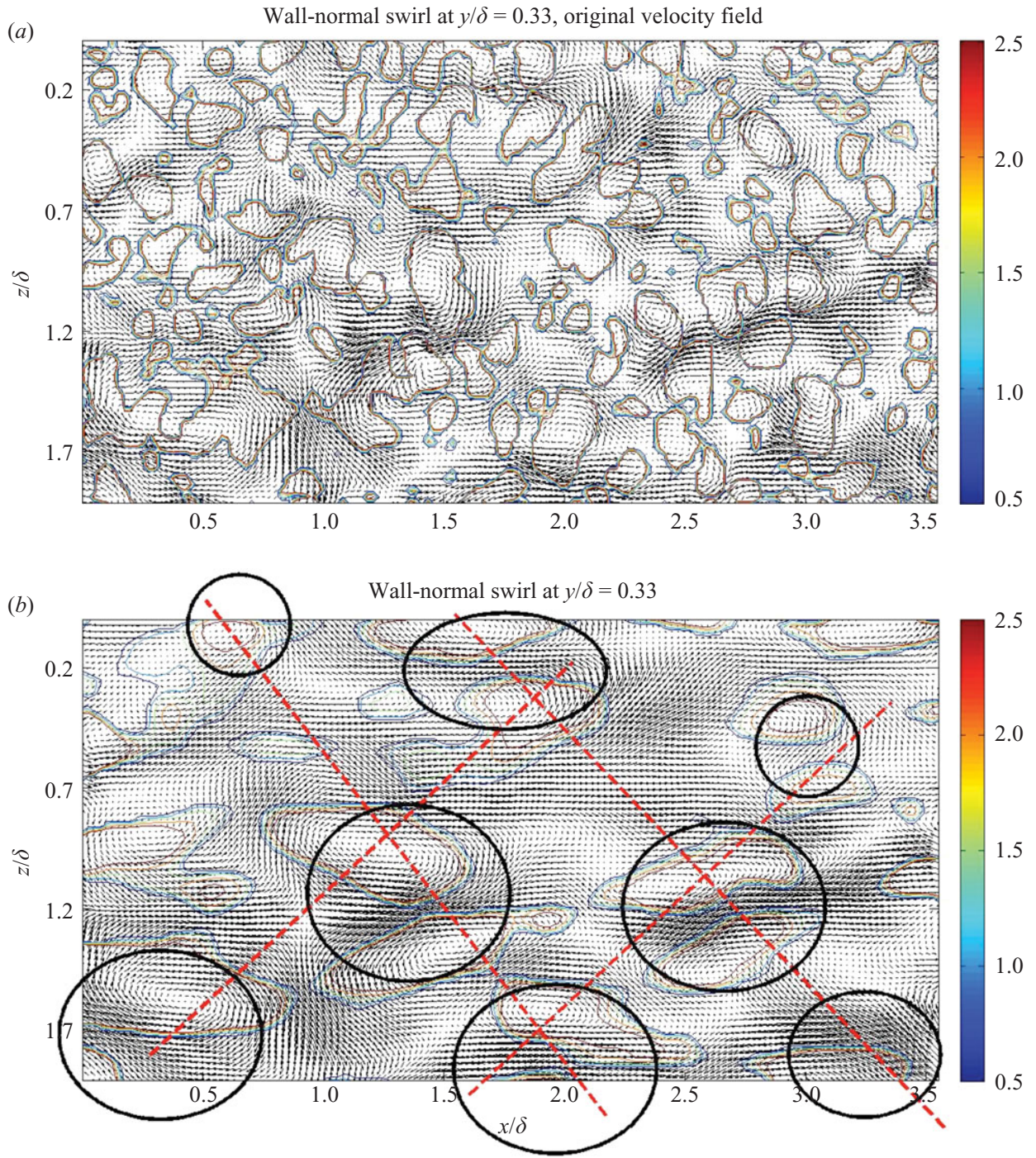

FIGURE 13 . Velocity vectors in the $x, z$ plane at $y / \delta=0.33$ and wall-normal swirl $\lambda_{c i, y}$ contours. (a) Original velocity field; $(b)$ low-pass filtered velocity field. Hairpin and cane vortex signatures are indicated by the circles. The dashed lines show alignment of the hairpins along diagonal lines.
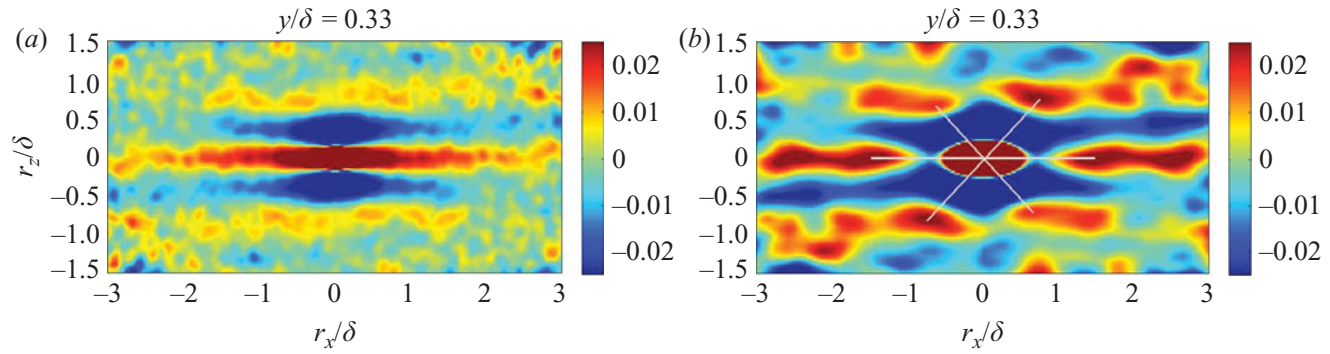

FIGURE 14. Autocorrelation coefficient of the directional wall-normal swirl $\lambda_{c i, y} \cdot \operatorname{sign}\left(\omega_{y}\right)$ in a plane parallel to the wall. (a) Original velocity field. (b) Low-pass filtered velocity field. 
The diagonal alignment of the large-scale hairpins is also found in the autocorrelation of wall-normal swirl strength $\lambda_{c i, y} \cdot \operatorname{sign}\left(\omega_{y}\right)$ in a plane parallel to the wall (figure 14a). Positive correlation peaks are found in the streamwise direction at $1.5 \delta$ distance from the DC peak and along the $45^{\circ}$ diagonals at approximately $0.8 \delta$ distance from the DC peak in both spanwise and streamwise direction (indicated by the grey lines). This means that starting from a wall-normal vortex, on average co-rotating wall-normal vortices exists at these relative positions, as is approximately the case in the instantaneous result of figure 13 . The level of the secondary correlation peak on the diagonal is 0.02 with an uncertainty of $10 \%$, which has been estimated from the differences in the autocorrelation function evaluated for subsets of the full dataset. This value of the correlation coefficient may appear small and insignificant, but is in fact of the same order as the secondary peak in the streamwise direction (approximately 0.03 ). Therefore, the diagonal pattern may be considered as important as the average streamwise alignment, which is associated to the very long lowspeed zones in the outer region of the turbulent boundary layer. The latter is a widely reported feature that is regarded relevant in the literature at present. The autocorrelation function also shows local minima adjacent to maxima in spanwise direction at $0.4 \delta$ distance, most noticeably on either side of the DC peak, which can be explained by the large-scale hairpins having counter-rotating necks $0.4 \delta$ apart (see figure 11). In contrast, the autocorrelation of $\lambda_{c i, y} \cdot \operatorname{sign}\left(\omega_{y}\right)$ based on the original unfiltered velocity fields (figure 14a) shows no obvious diagonal alignment, but displays only a strong streamwise alignment. This difference demonstrates that a filtering procedure is necessary to study the large-scale motions effectively, i.e. without the smaller scales affecting the statistics or dominating the visualization of swirling motion.

To check that the structure in the autocorrelation map is not introduced by the filtering, the procedure has been applied to random velocity distributions. The autocorrelation of wall-normal swirl in that case returns to zero away from the DC peak at distances greater than the kernel size, which verifies that the observed pattern is not due to the filter.

At larger distances from the wall the intermittency affects the autocorrelation. Consequently a larger number of samples is required for convergence, which unfortunately is not available at present. However, the instantaneous realizations, like the one shown in figure 12(b), do reveal a staggered arrangement of large-scale hairpin heads and necks above $y / \delta=0.6$, from which it is concluded that the diagonal pattern extends to higher elevations in the boundary layer.

The existence of a very large-scale spanwise or diagonal flow organization is rarely commented upon in literature. In transitional flow, based on flow visualizations, Perry, Lim \& Teh (1981) have described the initial stages of a turbulent spot using a staggered arrangement of vortex filaments. For a very low Reynolds number $\left(R e_{\theta}=700\right)$ Delo, Kelso \& Smits (2004) have suggested a similar diagonal alignment of dye structures observed in their incompressible turbulent boundary layer. However, the statistical support by the autocorrelation functions in their work contains some uncertainties. Above $y / \delta=1$, for instance, the result is not converged and consequently multiple orientations are detectable. Furthermore, below $y / \delta=0.7$ the primary orientation of the structures in their correlation map falls within a narrow range of approximately $10^{\circ}$ with the streamwise direction, which is decreasing further as the wall is approached and is clearly different from the $45^{\circ}$ diagonal. It is, therefore, well possible, that the smaller $10^{\circ}$ angle reflects another phenomenon like the meandering of the low-speed zones causing a skewing with respect to the streamwise direction (Hutchins \& Marusic 
2007, and also visible in figure 2). Because of the variations in the relative orientations and distances between adjacent large-scale vortices in the individual snapshots, as also noted in Delo et al. (2004), a statistic consistent with the instantaneous observations of a diagonal pattern is desirable. This has been possible to some extent in the present investigation. Moreover, the interpretations of the previously reported smoke and dye visualizations in terms of fluid motions are complicated and they have important subjective components. Still, the instantaneous transitional and the very low Reynolds number patterns appear similar to our high-Reynolds-number pattern, and this provides some reason to believe that the diagonal organization of the large-scale hairpins observed in this study could be found in a wider range of flow conditions.

\section{Discussion}

In the above analysis of the experimental results two scales of hairpin vortices have been identified: the large-scale hairpin $(\S 6)$ with a typical width of $0.4 \delta$ and a smaller scale, $0.25 \delta$ wide hairpin $(\S 5)$. The latter is at least four times stronger than the large-scale hairpin in terms of the peak $Q$ values. Both types of vortices are found near low-speed zones, and therefore the question arises how these vortices are organized relative to each other.

A possible answer to this question is provided by the short $\delta$-scale low-speed zones occasionally observed in the velocity volumes. An example is presented in figure 15, where the low-speed zone (blue) extends upstream of a single large-scale cane vortex with only part of the neck (magenta) inside the measurement volume. It must be noted that the low-speed zone extends down to $x / \delta=2.6$, where $u$ is just above the applied threshold for the blue isosurface and is approaching the local average velocity. The lower threshold is used here for visualization purpose; that is, not to obscure the vortices. Over the low-speed zone several smaller scale arch and cane shaped vortices (green) are seen. The most downstream vortices (denoted 'canes' in figure 15) appear slightly larger and more distorted compared to the upstream arches. This result suggests that the smaller scales occur or are formed on the low-speed zone associated with the neck and leg of the large-scale cane (or hairpin).

The findings are summarized in the conceptual sketch of figure 16, in which the large-scale hairpins are aligned in streamwise direction and along the diagonals as suggested by the autocorrelations function (figure 14). Near the wall $(y / \delta<0.5)$, the neck and legs of these large-scale hairpins create long low-speed zones, as observed in the conditional eddy of figure 11, which then connect to form the very long low-speed zones reported in literature (e.g. Kim \& Adrian 1999; Ganapathisubramani et al. 2007a; Hutchins \& Marusic 2007). The smaller scale hairpins in turn are located on top or inside the low-speed zones.

Farther from the wall, say $y / \delta>0.5$, shorter hairpin packets are found near the heads and necks of the large-scale hairpins. These packets generally do not connect in streamwise direction and therefore do not form very long zones of uniform low velocity. Consequently, the streamwise coherence length decreases with distance from the wall. The presence of hairpin packets in each snapshot at all three heights investigated suggest that several uniform (low-) momentum zones (Meinhart \& Adrian 1995) or packets stacked on top of each other (figure 25 in Adrian et al. 2000) can exist simultaneously within the envelope of retarded flow induced by the large-scale hairpins. In this picture the uniform momentum zone near the wall correspond to 


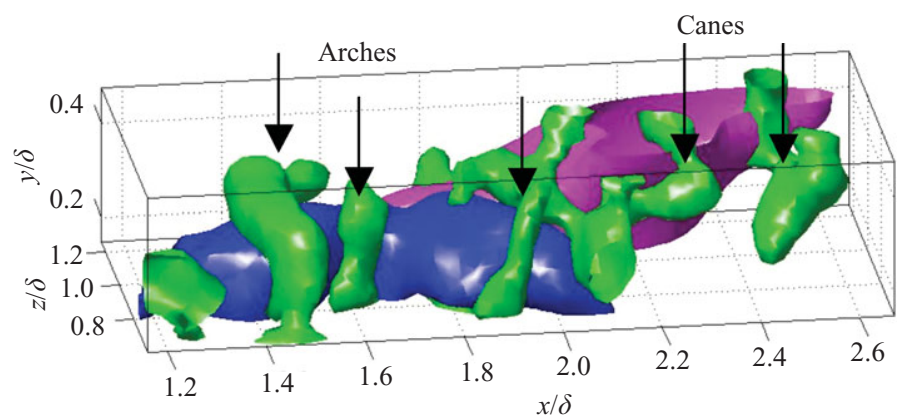

FIGURE 15. Large-scale and smaller scale vortices observed around a short $1.4 \delta$ low-speed zone. Blue represents part of a low-speed zone $\left(u<0.84 U_{e}\right)$, green is a $Q$ isosurface indicating vortical motion in the unfiltered velocity and magenta is an isosurface of the wall-normal swirl in the low-pass filtered velocity, which indicates the neck of a large-scale cane.

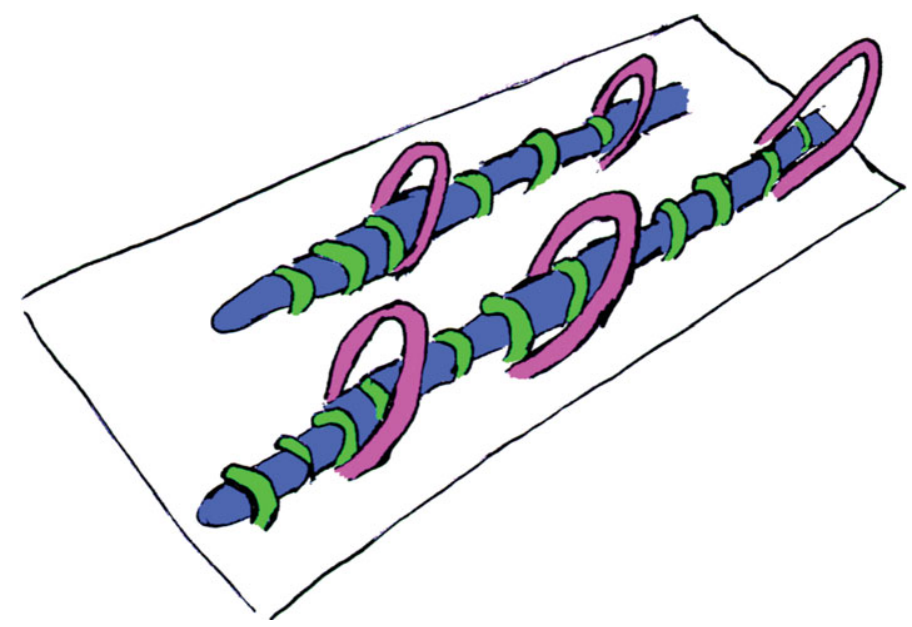

FIGURE 16. Conceptual sketch of the large-scale (magenta) and smaller scale hairpins (green) around the low-speed zones (blue).

the observed very long low-speed zones (figure 16), whereas the uniform momentum zones further from the wall are the shorter hairpin packets as seen in figure 3 .

\section{Conclusions}

The instantaneous, three-dimensional velocity distribution in a supersonic turbulent boundary layer with $R e_{\theta}=34,000, M_{e}=2.1$ and thickness $\delta=20 \mathrm{~mm}$ has been measured by tomographic PIV. The results present a quantitative visualization of the flow structures in the outer layer from $0.15 \delta$ to $0.89 \delta$ distance to the wall. The individual measurement volume ranges over $3.5 \delta \times 0.32 \delta \times 1.8 \delta$ in streamwise, wall-normal and spanwise direction and contains $142 \times 14 \times 77$ velocity vectors.

The instantaneous velocity fields reveal long low-speed zones commonly extending beyond the measurement volume $(>3.5 \delta)$ similar to results from previous studies. Over these zones hairpin vortices are observed, aligned in streamwise direction forming the so-called packets. Statistical support for the existence of hairpins at the present Reynolds and Mach number is provided by the conditional averaged eddy (figure 5). 
The conditional eddy further suggests that the hairpins are contained in a wider and much larger region of relatively low velocity.

The individual supersonic hairpins and the conditional eddy have been compared with eddies obtained in an incompressible low-speed turbulent boundary layer. Both the shape and the size of the eddies are found to be very similar for the two flow cases when scaled with the outer length scale (the boundary layer thickness $\delta$ ), which suggests universality of these structures.

Additionally, the velocity fields have been low-pass filtered to reveal $1.0 \delta$ long streamwise vortices and signatures of large-scale hairpins $0.4 \delta$ wide. Large-scale hairpin heads have also been observed at larger distance from the wall $(y / \delta>0.6)$ likely corresponding to the mentioned signatures closer to the wall. These largescale hairpin structures are reminiscent of turbulent bulges as for example described by Blackwelder \& Kovasznay (1972) and Falco (1977). Furthermore, they display a preferential alignment in streamwise direction (at $1.5 \delta$ spacing) and along the diagonals at $45^{\circ}$ with streamwise direction in the plane parallel to the wall. Evidence for this staggered arrangement of the large-scale hairpins is found in both instantaneous realizations as well as in the statistics, i.e. the autocorrelation of wall-normal swirl in the plane parallel to the wall.

The streamwise alignment of the large-scale hairpins results in very long regions of low velocity. These regions are $0.4 \delta$ wide as determined from a conditional averaging, which is consistent with the observed low velocity imprint in conditional eddy corresponding to the smaller scale hairpin. Furthermore, both scales of hairpins are found near the same low-speed zones in the instantaneous volumes. It is therefore believed that the smaller hairpins are contained within the envelope of low-speed flow induced by the large-scale hairpins.

This work is supported by the Dutch Technology Foundation STW under the 'VIDI Vernieuwingsimpuls' programme grant DLR.6198. LaVision GmbH is gratefully acknowledged for providing the camera system and Bernd Wieneke contributed in the data processing providing the volume self-calibration procedure. Furthermore, the help of Dirk Jan Kuik and Ray Humble during the measurements is greatly appreciated. Part of the work was carried out when G. E. Elsinga was visiting Arizona State University with the support of the Ira A. Fulton endowment.

\section{Appendix. Frequency response of the regression filter}

The spatial frequency response of the second-order regression filter of (3.3) is illustrated here by considering a one-dimensional velocity field before filtering $V(x)$ of the form

$$
V(x)=u_{0} \sin (2 \pi x / \lambda)
$$

and a three-dimensional distribution $V(x, y, z)$ of the form

$$
V(x, y, z)=u_{0} \sin (2 \pi x / \lambda) \sin (2 \pi y / \lambda) \sin (2 \pi z / \lambda)
$$

where $\lambda$ is the wavelength and $u_{0}$ is the amplitude. When comparing the filtered velocity distribution with the actual velocity a velocity modulation coefficient may be defined as the ratio of the peak velocity after and before filtering $u / u_{0}$. The modulation coefficient for the velocity gradient $u_{x} / u_{x, 0}$ is defined in a similar manner. The resulting modulation coefficients for different wavelengths are shown in figures 17 and 18 for the one-dimensional and three-dimensional case respectively. In the plots the wavelength length $\lambda$ has been normalized by the filter width or kernel size $W f$. 

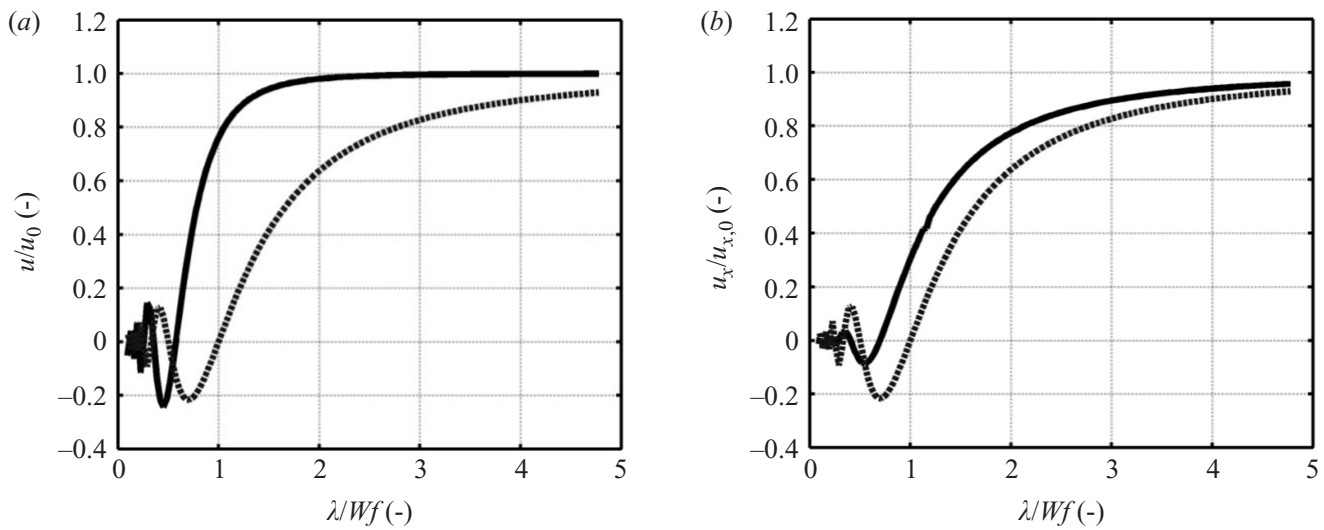

FIGURE 17. Modulation of the one-dimensional velocity field $(a)$ and velocity gradient $(b)$ by the second-order regression (solid line) and moving average filter (dashed line) as a function of wavelength.
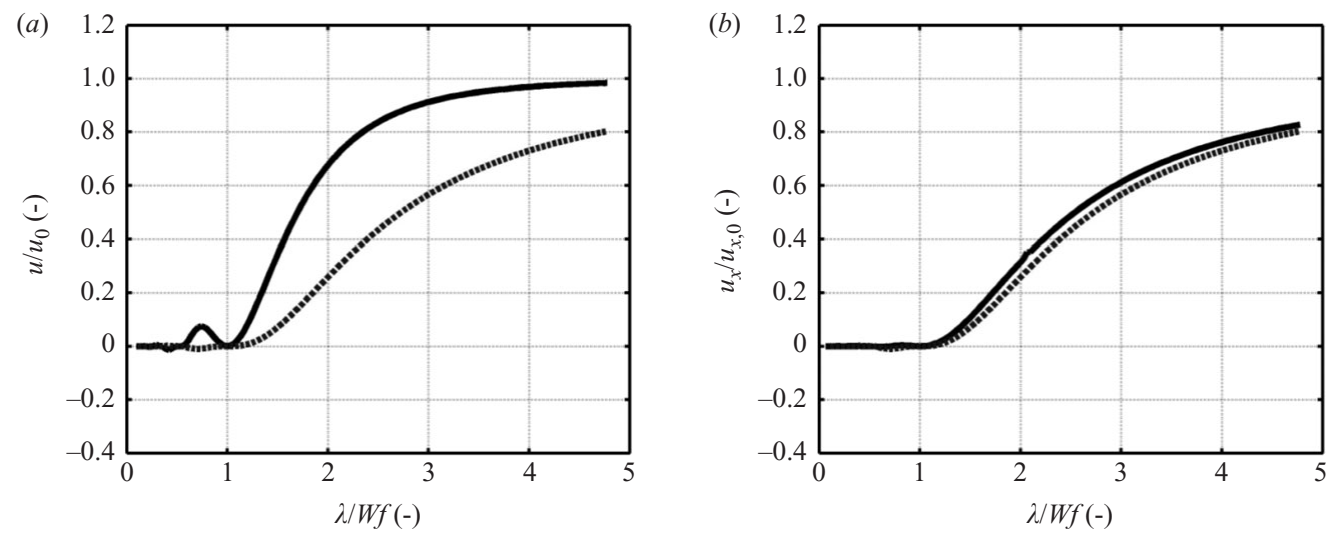

FIGURE 18. Modulation of the three-dimensional velocity field $(a)$ and velocity gradient $(b)$ by the second-order regression (solid line) and moving average filter (dashed line) as a function of wavelength.

Moreover, the moving average filter response is included in the plot as a reference. For the one-dimensional velocity distribution minor amplitude modulation $\left(u / u_{0}>0.9\right)$ is observed for wavelengths $\lambda / W f>1.3$ (figure 17a). The modulation increases quickly (decreasing $u / u_{0}$ ) with decreasing wavelength when the wavelength is smaller than the filter width $(\lambda / W f<1)$. Furthermore, small negative values of $u / u_{0}$ are found near $\lambda / W f=0.5$, which is indicative of the filtered velocity being out of phase with the actual velocity. This is, however, of no consequence when $W f$ is chosen as the cross-correlation window size, since these frequencies are not resolved by PIV.

Compared to the one-dimensional case, the modulation coefficient at a given wavelength is lower for the three-dimensional velocity field (figure 18a), where $u / u_{0}>0.9$ for wavelengths $\lambda / W f>2.9$. Another clear difference is the absence of negative $u / u_{0}$ peak near $\lambda / W f=0.5$. Although not shown, a two-dimensional velocity distribution has been considered also. Its wavelength response is in between the oneand three-dimensional result, as expected, with $u / u_{0}>0.9$ for wavelengths $\lambda / W f>2.3$. 
Finally, the velocity gradient modulation for the regression (figures $17 a$ and 18a) is found to be very similar to the moving average filter modulation, especially for the three-dimensional case.

\section{REFERENCES}

Adrian, R. J. 1996 Stochastic estimation of the structure of turbulent fields. In Eddy Structure Identification (ed. J. P. Bonnet), pp. 145-195. Springer-Verlag.

Adrian, R. J. 2007 Hairpin vortex organization in wall turbulence. Phys. Fluids 19, 041301.

Adrian, R. J., Meinhart, C. D. \& Tomkins, C. D. 2000 Vortex organization in the outer region of the turbulent boundary layer. J. Fluid Mech. 422, 1-54.

Balakumar, B. J. \& Adrian, R. J. 2007 Large- and very large-scale motions in channel and boundary-layer flows. Phil. Trans. R. Soc. A 365, 665-681.

Blackwelder, R. F. \& Kovasznay, L. S. G. 1972 Time scales and correlations in a turbulent boundary layer. Phys. Fluids 15, 1545.

Chakraborty, P., Balachandar, S. \& Adrian, R. J. 2005 On the relationship between local vortex identification schemes. J. Fluid Mech. 535, 189-214.

Christensen, K. T. \& Adrian, R. J. 2001 Statistical evidence of hairpin vortex packets in wall turbulence. J. Fluid Mech. 431, 433-443.

Delo, C. J., Kelso, R. M. \& Smits, A. J. 2004 Three-dimensional structure of a low-Reynoldsnumber turbulent boundary layer. J. Fluid Mech. 512, 47-83.

ElsingA, G. E. 2008 Tomographic particle image velocimetry and its application to turbulent boundary layers. Ph.D. dissertation, Delft University of Technology, Delft (http://repository.tudelft.nl/file/1003861/379883).

Elsinga, G. E., Kuik, D. J., van Oudheusden, B. W. \& Scarano, F. 2007 Investigation of the three-dimensional coherent structures in a turbulent boundary layer. In Forty-fifth AIAA Aerospace Sciences Meeting, Reno, NV, AIAA-2007-1305.

Elsinga, G. E., van Oudheusden, B. W. \& Scarano, F. $2006 a$ Experimental assessment of tomographic-PIV accuracy. In Thirteenth Intl Symp. on Laser Techniques to Fluid Mech., Lisbon, Portugal, Paper 20.5.

Elsinga, G. E., Scarano, F., Wieneke, B. \& van Oudheusden, B. W. $2006 b$ Tomographic particle image velocimetry. Exp. Fluids 41, 933-947.

FALCO, R. E. 1977 Coherent motions in the outer region of turbulent boundary layers. Phys. Fluids 20, S124-S132.

Ganapathisubramani, B., Clemens, N. T. \& Dolling, D. S. 2006 Large-scale motions in a supersonic turbulent boundary layer. J. Fluid Mech. 556, 271-282.

Ganapathisubramani, B., Clemens, N. T. \& Dolling, D. S. 2007 a Effects of upstream boundary layer on the unsteadiness of shock-induced separation. J. Fluid Mech. 585, 369-394.

Ganapathisubramani, B., Clemens, N. T. \& Dolling, D. S. $2007 b$ Effects of upstream coherent structures on low-frequency motion of shock-induced turbulent separation. In Forty-fifth AIAA Aerospace Sciences Meeting, Reno, NV, AIAA-2007-1141.

Ganapathisubramani, B., Longmire, E. K. \& Marusic, I. 2003 Characteristics of vortex packets in the turbulent boundary layer. J. Fluid Mech. 478, 35-46.

GARG, S. \& SetTles, G. S. 1998 Measurements of a supersonic turbulent boundary layer by focusing schlieren deflectometry. Exp. Fluids 25, 254-264.

Guala, M., Hommema, S. E. \& Adrian, R. J. 2006 Large-scale and very-large-scale motions in turbulent pipe flow. J. Fluid Mech. 554, 521-542.

Head, M. R. \& Bandyopadhyay, P. 1981 New aspects of the turbulent boundary layer structure. J. Fluid Mech. 107, 297-338.

Humble, R. A., Elsinga, G. E., Scarano, F. \& van Oudheusden, B. W. 2009 Three-dimensional unsteady flow organization of shock wave/turbulent boundary layer interaction. J. Fluid Mech. 622, 33-62.

Humble, R. A., Scarano, F. \& van Oudheusden, B. W. 2007 Particle image velocimetry measurements of shock wave/turbulent boundary layer interaction. Exp. Fluids 43, 173183. 
Humble, R. A., Scarano, F., Van Oudheusden, B. W. \& Tuinstra, M. 2006 PiV measurements of Shock Wave/Turbulent Boundary Layer Interaction. In Thirteenth Intl Symp. on Laser Techniques to Fluid Mechanics, Lisbon, Portugal, Paper 14.2.

Hunt, J. C. R., Wray, A. A. \& Moin, P. 1988 Eddies, stream, and convergence zones in turbulent flows. Tech. Rep. Report CTR-S88. Centre for Turbulence Research, pp. 193-208.

Hutchins, N. \& Marusic, I. 2007 Evidence of very long meandering features in the logarithmic region of turbulent boundary layers. J. Fluid Mech. 579, 1-28.

Kim, K. C. \& Adrian, R. J. 1999 Very large-scale motion in the outer layer. Phys. Fluids 11, 417-422.

Kline, S. J., Reynolds, W. C., Schraub, F. A. \& Runstadler, P. W. 1967 The structure of turbulent boundary layers. J. Fluid Mech. 30, 741-773.

Martin, M. P. 2007 Direct numerical simulation of hypersonic turbulent boundary layers. Part 1. Initialization and comparison with experiments. J. Fluid Mech. 570, 347-364.

Meinhart, C. D. \& Adrian, R. J. 1995 On the existence of uniform momentum zones in a turbulent boundary layer. Phys. Fluids 7, 694-696.

Perry, A. E., Lim, T. T. \& Teh, E. W. 1981 A visual study of turbulent spots. J. Fluid Mech. 104, $387-405$.

Ringuette, M. J., Wu, M. \& Martin, M. P. 2008 Coherent structures in direct numerical simulation of turbulent boundary layers at Mach 3. J. Fluid Mech. 594, 59-69.

Robinson, S. K. 1991 Coherent motions in the turbulent boundary layer. Annu. Rev. Fluid Mech. 23, 601-639.

SavitzKy, A. \& Golay, M. J. E. 1964 Smoothing and differentiation of data by simplified least squares procedures. Anal. Chem. 36, 1627-1639.

Scarano, F. \& Riethmuller, M. L. 2000 Advances in iterative multigrid PIV image processing. Exp. Fluids 29, 51-60.

SchriJer, F. F. J. \& Scarano, F. 2007 Particle slip compensation in steady compressible flows. Seventh Intl Symp. on Particle Image Velocimetry, Rome, Italy.

Schröder, A., Geisler, R., Elsinga, G. E., Scarano, F. \& Dierksheide, U. 2007 Investigation of a turbulent spot and a tripped turbulent boundary layer using time-resolved tomographic PIV. Exp. Fluids 44, 305-316.

Smits, A. J. \& Dussauge, J. P. 2006 Turbulent shear layers in supersonic flow, 2nd edn. Springer.

Smits, A. J., Hayakawa, K. \& Muck, K. C. 1983 Constant temperature hot-wire anemometry practice in supersonic flows; Part 1. The normal wire. Exp. Fluids 1, 83-92.

Smith, M. W. \& Smits, A. J. 1995 Visualization of the structure of supersonic turbulent boundary layers. Exp. Fluids 18, 288-302.

Spina, E. F., Donovan, J. F. \& Smits, A. J. 1991 On the structure of high-Reynolds-number supersonic turbulent boundary layers. J. Fluid Mech. 222, 293-327.

Spina, E. F., Smits, A. J. \& Robinson, S. K. 1994 The physics of supersonic boundary layers. Annu. Rev. Fluid Mech. 26, 287-319.

Stanislas, M., Perret, L. \& Foucaut, J. M. 2008 Vortical structures in the turbulent boundary layer: a possible route to a universal representation. J. Fluid Mech. 602, 327-382.

Theodorsen, T. 1952 Mechanism of turbulence. In Proceedings of Second Midwestern Conference on Fluid Mechanics, Ohio State University, Columbus, OH, pp. 1-19.

Tomkins, C. D. \& Adrian, R. J. 2003 Spanwise structure and scale growth in turbulent boundary layers. J. Fluid Mech. 490, 37-74.

WiENEKE, B. 2008 Volume self-calibration for 3D particle image velocimetry. Exp. Fluids 45, 549-556.

Zhou, J., Adrian, R. J., Balachandar, S. \& Kendall, T. M. 1999 Mechanisms for generating coherent packets of hairpin vortices in channel flow. J. Fluid Mech. 387, 353-396. 